\title{
Model order reduction in hyperelasticity: a Proper Generalized Decomposition approach
}

\author{
Siamak Niroomandi ${ }^{1}$, Icíar Alfaro ${ }^{1}$, David González ${ }^{1}$, \\ Elías Cueto $^{1, *}$, Francisco Chinesta ${ }^{2}$ \\ ${ }^{1}$ Aragón Institute of Engineering Research (I3A). Universidad de Zaragoza. \\ Edificio Betancourt. María de Luna, s.n. 50018 Zaragoza, Spain. \\ ${ }^{2}$ EADS Corporate Foundation International Chair. École Centrale de Nantes. \\ 1, Rue de la Noë. 44300 Nantes, France.
}

\begin{abstract}
SUMMARY
This paper deals with the extension of Proper Generalized Decomposition (PGD) methods to non-linear problems, in particular to hyperelasticity. Among the different approaches that can be considered for the linearization of the doubly-weak form of the problem, we have implemented a new one that uses Asymptotic Numerical Methods in conjunction with PGD to avoid complex consistent linearization schemes necessary in Newton strategies. This approach results in an approximation of the problem solution in the form of a series expansion. Each term of the series is expressed as a finite sum of separated functions. The advantage of this approach is the presence of only one tangent operator, identical for every term in the series. The resulting approach has proved to render very accurate results that can be stored in the form of a meta-model in a very compact format. This opens the possibility to use these results in real-time, reaching $\mathrm{kHz}$ feedback rates, or to be used in deployed, handheld devices such as smartphones and tablets. Copyright (C) 2012 John Wiley \& Sons, Ltd.
\end{abstract}

KEY WORDS: Proper Generalized Decomposition, Separated representations, Asymptotic Numerical Methods, model order reduction, real time simulation, hyperelasticity

\section{Contents}

\section{Introduction 2}

2 Formulation of the problem under the PGD point of view 4

2.1 Computation of $S(s)$ assuming $R(\boldsymbol{x})$ is known . . . . . . . . . . . . 5

2.2 Computation of $R(\boldsymbol{x})$ assuming $S(s)$ is known . . . . . . . . . . . . . . 6

3 A brief review of the Asymptotic Numerical Method

\footnotetext{
*Correspondence to: Elías Cueto. Universidad de Zaragoza. Edificio Betancourt. María de Luna, s..n. E-50018 Zaragoza, Spain. e-mail: ecueto@unizar.es

Contract/grant sponsor: Spanish Ministry of Economy and Innovation; contract/grant number: CICYT-DPI2011-27778-C02-01 
4 A combined PGD-ANM approach to hyperelasticity 9

5 Numerical examples $\quad \mathbf{1 0}$

5.1 Kirchhoff-Saint Venant material . . . . . . . . . . . . . . . . . . . . 10

$5.1 .1 \quad$ Pinched cube . . . . . . . . . . . . . . . . . . . . . 10

5.1 .2 Beam bending . . . . . . . . . . . . . . . . 11

5.1 .3 Palpation of the liver . . . . . . . . . . . . . . . 12

5.2 Neo-hookean behavior . . . . . . . . . . . . . . . . . . 13

5.2 .1 Neo Hookean beam under bending . . . . . . . . . . . . . . . . . . . . . . 19

5.2 .2 Palpation of a neo-hookean liver . . . . . . . . . . . . . . . . 19

6 Conclusions 22

I Derivation of the tangent stiffness matrix for the neo-hookean case 23

1. Introduction

Proper Orthogonal Decomposition (POD) methods have had a tremendous importance in many branches of applied sciences and engineering, where they are known under a variety of names. Starting from the pioneer works by Karhunen [23] and Loève [29] (also Lorenz [30]), POD methods, also known as Principal Component Analysis, subspace tracking, among other names, have been applied to a wide range of problems, from chemical engineering [37] to real time visualization, possibly with haptic response [7], structural mechanics [22] [24] or virtual surgery [32] [44] [43].

POD rely upon the computation of the solution to surrogate problems, the so-called snapshots. The most typical structure of these snapshots, obtained as an eigenvalue problem through a minimization process, is then used as a basis for subsequent problem solving. Among the problems related to this approach one can cite the need for an important number of snapshots. These snapshots should be generated by solving problems somewhat similar to the one at hand, but maybe for slight changes in geometry or boundary conditions. The so-called a priori Proper Orthogonal Decomposition [40] [41] [42] constitutes one of the first attempts to aviod the costly generation of snapshots by starting from scratch and adding Krylov subspaces to the basis of the reduced model.

Another difficulty on the use of POD-based model order reduction is related to the need of recomputing the original system of equations in non-linear problems (even if it will not be solved) in order to update tangent stiffness matrices. This prevents the method to provide as competitive costs as one would expect from the limited size of the resulting systems of equations. This problem has generated recently quite an active area of research. As notable contributions, one can cite the Empirical Interpolation Method [8] or its discrete counterpart [13]. A somewhat related approach can be found in [31].

On the other hand, non-linear POD methods (those designed for data lying on a manifold) are always difficult to develop. See [7] [45] [35] [33] to name a few. Interpolation among reduced models continues to be an issue too [6] [35].

Proper Generalized Decomposition (PGD) methods, on the contrary, arose recently as a generalization of POD techniques. PGD roots can be traced back to the pioneer work by P. Ladeveze on the LATIN method [25] and, particularly, the so-called radial loading approximation scheme, in which a separated space-time approximation of the displacement in structural mechanics problems 
was used. Independently, the method was re-invented in the framework of problems defined in high dimensional state spaces [5] [4]. It was then soon realized that PGD methods can be considered as a generalization of POD, in which the basis are computed on the fly without no previous snapshot. Instead, the essential field is approximated as a finite sum of separable functions, very much like the space-time structure of the radial approximation within the LATIN method. For recent surveys on PGD methods, the reader is referred to [14] [15] [26]. Some of the nice properties of POD are lost, however. For instance, optimality of the POD basis is no more guaranteed, although convergence properties have been demonstrated recently [28] and error estimators have also been proposed [3] [27].

When compared to existing model order reduction techniques, such as the ones based on POD, for instance, PGD provides the modes a priori, i.e., without the need to compute snap- shots of the complete model. These snapshots would be extremely costly to generate for a problem defined in high dimensional phase spaces, where the sampling of the space is not obvious. With respect to the technique introduced in [7] on the contrary, it is stated that "Deformation basis generation is a hard open problem in solid mechanics, and there exist no algorithms for automatic proven-quality global deformation basis generation under general forcing." This is precisely where PGD comes into play. The method here proposed provides with a suitable set of reduced basis a priori, without the need to compute costly snapshots. In [7], two different method were chosen to generate the basis, one based upon standard modal analysis which includes derivatives of the eigenmodes. The second one is basically a POD procedure in which the modes are extracted from a linear simulation and successively enriched offline. However, although in this reference it is stated that the method is general and can be applied to constitutive models other than Kirchhoff Saint Venant (which is low practical interest due to its well-known instabilities in compression due to lack of convexity of the strain energy functional), we believe that it is difficult to generalize for state of the art constitutive hyperelastic models. Up to our knowledge, it has not been generalized yet.

One key aspect in the rapid development of PGD methods is related to the fact that PGD can be seen as both an efficient technique for high dimensional problems and as an a priori model order reduction technique. This opened the door to re-interpreting parametric problems as high dimensional ones, just by considering parameters as new dimensions of the state space of the model [38] [19] [20].

This new point of view offered new and interesting insights in many branches of applied science and engineering. It is now envisaged how by looking at a parametric problem from a multidimensional framework gives rise to important savings in simulation time. Furthermore, it has been observed how important advantages could be obtained by formulating standard problems as high dimensional ones. For instance, formulating plate and shell problems as three-dimensional ones, but with twodimensional computational complexity [9] has revealed very interesting insights on a general finite element formulation beyond Kirchhoff and Reissner-Mindlin plates.

In this work we explore the possibilities of PGD methods applied to fast (real time) simulation of hyperelastic solids. For a related reference, the interested reader can also consult [39]. It will be shown how a multi-dimensional formulation of the problem, in which the displacement is expressed as to be dependent on both the physical coordinates and the position and orientation of the applied loads (thus, defined in $\mathbb{R}^{9}$ ), opens the door to simulations with feedback rates on the order of one $\mathrm{kHz}$. The developed strategy comprises an off-line computation strategy, in which a high-dimensional problem is solved. This solution provides in fact a sort of meta-model that can be stored in a very compact form. Then, an on-line simulation strategy is developed that solves the meta-model at impressive feedback rates, even on handheld devices.

But the price to pay in this case is the complex non-linear structure of the weak form of the problem, as will be shown in Section 2. Consistent linearization of PGD weak forms continues to be an issue 
in our community. Previous works include explicit linearizations [34]. In this work we introduce a new approach by combining a PGD formulation of the problem with an asymptotic expansion of the displacement. The use of asymptotic expansions in the search of complex non-linear equilibrium paths of structures is due to Potier-Ferry and coworkers [16] [1] [12] [17]. This gives rise to a formulation in which only one tangent operator is present, the same for all the terms of the expansion, and where the non-linearities are translated to the right-hand-side (force) term of the matrix form of the problem. The resulting technique provides results, as will be shown, at $\mathrm{kHz}$ feedback rates for general hyperelastic models. This constitutes, in our opinion, a major advancement in the state of the art of reduced order models. Up to our knowledge, only techniques working with Kirchhoff-Saint Venant model were able to obtain such performance rates.

In Section 3 we review the basics of Asymptotic Numerical Methods (ANM) in their standard form, while in Section 4 we develop the combined PGD-ANM formalism and study how it provides a very convenient form for non-linear path following of hyperelastic solids and structures. In Section 5 we study the performance of the proposed technique by employing some classical benchmark tests and show how the resulting formalism can be employed advantageously to perform real time simulation.

\section{Formulation of the problem under the PGD point of view}

As mentioned before, one of the main advantages of using PGD for model order reduction purposes relies in the possibility of re-writing the model in a multi-dimensional framework. Consider, for simplicity, the static equilibrium equations of a general solid under small strain assumptions:

$$
\boldsymbol{\nabla} \cdot \boldsymbol{\sigma}+\boldsymbol{b}=\mathbf{0} \text { in } \Omega
$$

where $\boldsymbol{b}$ represents the volumetric forces applied to the body, subjected to the following boundary conditions

$$
\begin{gathered}
\boldsymbol{u}=\overline{\boldsymbol{u}} \text { on } \Gamma_{u} \\
\boldsymbol{\sigma} \boldsymbol{n}=\overline{\boldsymbol{t}} \text { on } \Gamma_{t}
\end{gathered}
$$

The standard weak form of the problem is obtained after multiplying both sides of Eq. (1) by an admissible variation of the displacement, $\boldsymbol{u}^{*}$, and integrating over the domain $\Omega$. In order to fully exploit the characteristics of PGD methods, following [38], we convert the equilibrium problem given by Eqs. (1)-(3) into a parametric one, by considering, again for the sake of simplicity in the exposition, that the load $\bar{t}$ is punctual, unitary, vertical, and is applied at a position $s \in \Gamma_{t}$ that acts as a parameter in the formulation. Note that this alternative formulation of the problem gives rise to a new one defined in $\mathbb{R}^{6}$, since $\boldsymbol{u}=\boldsymbol{u}(\boldsymbol{x}, \boldsymbol{s}) \in \Omega \times \bar{\Gamma}$, where $\bar{\Gamma} \subseteq \Gamma_{t}$ represents the portion of the boundary where the load can be applied.

If inertia terms in Eq. (1) were non-negligible, a possible approach that considers initial conditions as additional parameters has also been explored in [21].

In this spirit, an alternative (doubly) weak form of problem (1)-(3) consists in finding the displacement $\boldsymbol{u} \in \mathcal{H}^{1}(\Omega) \times L_{2}(\bar{\Gamma})$ such that for all $\boldsymbol{u}^{*} \in \mathcal{H}_{0}^{1}(\Omega) \times L_{2}(\bar{\Gamma})$ (see [34]):

$$
\int_{\bar{\Gamma}} \int_{\Omega}\left(\nabla_{s} \boldsymbol{u}^{*}\right)^{T} \boldsymbol{\sigma} d \Omega d \bar{\Gamma}=\int_{\bar{\Gamma}} \int_{\Gamma_{t 2}}\left(\boldsymbol{u}^{*}\right)^{T} \boldsymbol{t} d \Gamma d \bar{\Gamma}
$$

where $\Gamma=\Gamma_{u} \cup \Gamma_{t}$ represents the boundary of the solid, divided into essential and natural regions, and where $\Gamma_{t}=\Gamma_{t 1} \cup \Gamma_{t 2}$, i.e., regions of homogeneous and non-homogeneous, respectively, natural 
boundary conditions. In turn, we assume $t=e_{z} \delta(x-s)$, where $\delta$ represents the Dirac-delta function and $\boldsymbol{e}_{z}$ represents the unit vector along the $z$ direction, in this case. Note that a general-form load term does not include further complexity into this formulation. This Dirac-delta term should be regularized for computation purposes and approximated by:

$$
t_{j} \approx \sum_{i=1}^{n} f_{j}^{i}(\boldsymbol{x}) g_{j}^{i}(\boldsymbol{s})
$$

by simply performing a singular value decomposition of the load, for instance.

As explained before, PGD methods assume a separated representation of the unknown field (here, the displacement). This is the key ingredient of the method that allows solving efficiently high dimensional problems. The so-called curse of dimensionality associated to mesh-based solution of high dimensional problems is thus avoided by solving a sequence of low-dimensional problems in separated form. This was in fact the key ingredient in the space-time separated representation of the displacement in the LATIN method [25]. The PGD approach to the problem is characterized by the construction, in an iterative way, of an approximation to the solution in the form of a finite sum of separable functions. Assume that we have converged to a solution, at iteration $n$, of this procedure,

$$
u_{j}^{n}(\boldsymbol{x}, \boldsymbol{s})=\sum_{k=1}^{n} X_{j}^{k}(\boldsymbol{x}) \cdot Y_{j}^{k}(\boldsymbol{s}),
$$

where the term $u_{j}$ refers to the $j$-th component of the displacement vector, $j=1,2,3$.

The following term of this approximation, the $(n+1)$-th one, will look like

$$
u_{j}^{n+1}(\boldsymbol{x}, \boldsymbol{s})=u_{j}^{n}(\boldsymbol{x}, \boldsymbol{s})+R_{j}(\boldsymbol{x}) \cdot S_{j}(\boldsymbol{s}),
$$

where $\boldsymbol{R}(\boldsymbol{x})$ and $\boldsymbol{S}(\boldsymbol{s})$ are the sought functions that improve the approximation. In this same way, the admissible variation of the displacement will be given by

$$
u_{j}^{*}(\boldsymbol{x}, \boldsymbol{s})=R_{j}^{*}(\boldsymbol{x}) \cdot S_{j}(\boldsymbol{s})+R_{j}(\boldsymbol{x}) \cdot S_{j}^{*}(\boldsymbol{s}) .
$$

At this point several options are at hand so as to determine the new pair of functions $R_{j}$ and $S_{j}$. The most frequently used, due to both its ease of implementation and good convergence properties, in general, is a fixed-point alternating directions algorithm in which functions $R_{j}$ and $S_{j}$ are sought iteratively. We describe briefly the implementation of this algorithm.

\subsection{Computation of $S(\boldsymbol{s})$ assuming $R(\boldsymbol{x})$ is known}

In this case, following standard assumptions of variational calculus, we have

$$
u_{j}^{*}(\boldsymbol{x}, \boldsymbol{s})=R_{j}(\boldsymbol{x}) \cdot S_{j}^{*}(\boldsymbol{s}),
$$

or, equivalently, $\boldsymbol{u}^{*}(\boldsymbol{x}, \boldsymbol{s})=\boldsymbol{R} \circ \boldsymbol{S}^{*}$, where the symbol "॰" denotes the so-called entry-wise, Hadamard or Schur multiplication for vectors. Once substituted into Eq. (4), gives

$$
\begin{aligned}
\int_{\bar{\Gamma}} \int_{\Omega} \nabla_{s}\left(\boldsymbol{R} \circ \boldsymbol{S}^{*}\right): \mathbf{C}: \boldsymbol{\nabla}_{s}\left(\sum_{k=1}^{n} \boldsymbol{X}^{k} \circ \boldsymbol{Y}^{k}+\boldsymbol{R} \circ \boldsymbol{S}\right) d \Omega d \bar{\Gamma}= \\
\qquad \int_{\bar{\Gamma}} \int_{\Gamma_{t 2}}\left(\boldsymbol{R} \circ \boldsymbol{S}^{*}\right) \cdot\left(\sum_{k=1}^{m} \boldsymbol{f}^{k} \circ \boldsymbol{g}^{k}\right) d \Gamma d \bar{\Gamma},
\end{aligned}
$$


or, equivalently (we omit obvious functional dependencies)

$$
\begin{aligned}
& \int_{\bar{\Gamma}} \int_{\Omega} \nabla_{s}\left(\boldsymbol{R} \circ \boldsymbol{S}^{*}\right): \mathbf{C}: \nabla_{s}(\boldsymbol{R} \circ \boldsymbol{S}) d \Omega d \bar{\Gamma} \\
& =\int_{\bar{\Gamma}} \int_{\Gamma_{t 2}}\left(\boldsymbol{R} \circ \boldsymbol{S}^{*}\right) \cdot\left(\sum_{k=1}^{m} \boldsymbol{f}^{k} \circ \boldsymbol{g}^{k}\right) d \Gamma d \bar{\Gamma}-\int_{\bar{\Gamma}} \int_{\Omega} \boldsymbol{\nabla}_{s}\left(\boldsymbol{R} \circ \boldsymbol{S}^{*}\right) \cdot \mathcal{R}^{n} d \Omega d \bar{\Gamma},
\end{aligned}
$$

where $\mathcal{R}^{n}$ represents:

$$
\mathcal{R}^{n}=\mathbf{C}: \boldsymbol{\nabla}_{s} \boldsymbol{u}^{n} .
$$

All the terms depending on $x$ are known and hence we can compute all integrals over $\Omega$ and $\Gamma_{t 2}$ (support of the regularization of the initially punctual load) to derive an equation to compute $S(s)$.

\subsection{Computation of $R(\boldsymbol{x})$ assuming $S(\boldsymbol{s})$ is known}

Equivalently, in this case, we have

$$
u_{j}^{*}(\boldsymbol{x}, s)=R_{j}^{*}(\boldsymbol{x}) \cdot S_{j}(\boldsymbol{s}),
$$

which, once substituted into Eq. (4), gives

$$
\begin{aligned}
\int_{\bar{\Gamma}} \int_{\Omega} \boldsymbol{\nabla}_{s}\left(\boldsymbol{R}^{*} \circ \boldsymbol{S}\right): \mathbf{C}: \boldsymbol{\nabla}_{s}\left(\sum_{k=1}^{n} \boldsymbol{X}^{k} \circ \boldsymbol{Y}^{k}+\boldsymbol{R} \circ \boldsymbol{S}\right) d \Omega d \bar{\Gamma}= \\
\qquad \int_{\bar{\Gamma}} \int_{\Gamma_{t 2}}\left(\boldsymbol{R}^{*} \circ \boldsymbol{S}\right) \cdot\left(\sum_{k=1}^{m} \boldsymbol{f}^{k} \circ \boldsymbol{g}^{k}\right) d \Gamma d \bar{\Gamma} .
\end{aligned}
$$

In this case all the terms depending on $s$ (load position) can be integrated over $\bar{\Gamma}$, leading to a generalized elastic problem to compute function $\boldsymbol{R}(\boldsymbol{x})$.

This simple algorithm renders, in general, excellent convergence properties (see [15] and references therein).

While fixed-point approaches (see [15] and references therein) render in general excellent results, it is in general very difficult to perform consistent linearizations on Eqs. (11) and (14). In [34] some explicit algorithms have been tested to overcome this problem that, in general, work well. By this we mean that they provide errors, for reasonable pseudo-time step sizes, of the same order of those obtained by existing POD-based approaches such as [35] [33]. This level of error is, in general, higher than that of common engineering practice, but is nevertheless acceptable in the real-time simulation community [11].

In what follows a method that combines asymptotic expansions of the displacement and the second Piola- Kirchhoff stress tensor to avoid complex stiffness matrix update procedures is reviewed. The method, due to Potier-Ferry and coworkers [16] [1] [12] [17] [46], has been used in a variety of structural mechanics problems to follow complex equilibrium paths of solids and structures.

\section{A brief review of the Asymptotic Numerical Method}

For completeness, and following closely [46], we review here the basics of the Asymptotic Numerical Method applied to the simplest non-linear case, namely, Kirchhoff-Saint Venant solids (i.e., linear 
elastic solids undergoing large strains). Under a Lagrangian frame of reference we consider the displacement as given by

$$
\boldsymbol{x}=\boldsymbol{X}+\boldsymbol{u} .
$$

Following the notation in [17], we consider a linear and a non-linear term for the Green-Lagrange strain tensor, $\boldsymbol{E}$, in the form

$$
\boldsymbol{E}=\frac{1}{2}\left(\boldsymbol{F}^{T} \boldsymbol{F}-1\right)=\gamma_{l}(\boldsymbol{u})+\gamma_{n l}(\boldsymbol{u}, \boldsymbol{u})
$$

where $\boldsymbol{F}=\boldsymbol{\nabla} \boldsymbol{u}+\boldsymbol{I}$ is the gradient of deformation tensor and

$$
\begin{aligned}
\gamma_{l}(\boldsymbol{u}) & =\frac{1}{2}\left(\boldsymbol{\nabla}\left(\boldsymbol{u}^{T}\right)+\nabla(\boldsymbol{u})\right), \\
\gamma_{n l}(\boldsymbol{u}, \boldsymbol{u}) & =\frac{1}{2} \boldsymbol{\nabla}\left(\boldsymbol{u}^{T}\right) \boldsymbol{\nabla}(\boldsymbol{u}) .
\end{aligned}
$$

Hyperelastic materials are base on the assumption of a particular strain-energy function, $\Psi$. Then the second Piola-Kirchhoff stress tensor $S$ can thus be obtained by

$$
\boldsymbol{S}=\frac{\partial \Psi}{\partial \boldsymbol{E}}
$$

that is a symmetric tensor and is related to the first Piola-Kirchhoff stress tensor, $\boldsymbol{P}$, by $\boldsymbol{P}=\boldsymbol{F} \boldsymbol{S}$.

The equilibrium equation stated in the reference configuration looks like

$$
\boldsymbol{\nabla} \boldsymbol{P}+\boldsymbol{B}=\mathbf{0} \text { in } \Omega_{0}
$$

in which $\boldsymbol{B}$ is the body force. The boundary conditions of the body are defined by (we do not consider the case of follower loads for simplicity)

$$
\begin{gathered}
\boldsymbol{u}(\boldsymbol{X})=\overline{\boldsymbol{u}} \text { on } \Gamma_{u}, \\
\boldsymbol{P N}=\bar{\lambda} \overline{\boldsymbol{t}} \text { on } \Gamma_{t}
\end{gathered}
$$

where $N$ is the unit vector normal to $\Gamma=\partial \Omega_{0}, \bar{t}$ is an applied traction and $\bar{\lambda}$ is a loading parameter, equivalent to a pseudo-time, and ranging from 0 to 1 . The weak form of the problem is then given by

$$
\int_{\Omega_{0}} \boldsymbol{S}: \boldsymbol{E}^{*} d \Omega=\bar{\lambda} \int_{\Gamma_{t}} \overline{\boldsymbol{t}} \cdot \boldsymbol{u}^{*} d \Gamma \forall \boldsymbol{u}^{*} \in H^{1}(\Omega)
$$

where in the above equation $\boldsymbol{E}^{*}$ is expressed by

$$
\boldsymbol{E}^{*}=\frac{1}{2}\left[\boldsymbol{F}^{T} \boldsymbol{\nabla}\left(\boldsymbol{u}^{*}\right)+\nabla\left(\boldsymbol{u}^{*}\right)^{T} \boldsymbol{F}\right]=\gamma_{l}\left(\boldsymbol{u}^{*}\right)+\gamma_{n l_{S}}\left(\boldsymbol{u}, \boldsymbol{u}^{*}\right),
$$

where, in turn, $\gamma_{n l_{S}}\left(\boldsymbol{u}, \boldsymbol{u}^{*}\right)$ is defined by

$$
\gamma_{n l_{S}}\left(\boldsymbol{u}, \boldsymbol{u}^{*}\right)=\gamma_{n l}\left(\boldsymbol{u}, \boldsymbol{u}^{*}\right)+\gamma_{n l}\left(\boldsymbol{u}^{*}, \boldsymbol{u}\right) .
$$

The Kirchhoff-Saint Venant model is characterized by the energy function given by

$$
\Psi=\frac{\lambda}{2}(\operatorname{tr}(\boldsymbol{E}))^{2}+\mu \boldsymbol{E}: \boldsymbol{E}
$$


where $\lambda$ and $\mu$ are Lame's constants. The second Piola-Kirchhoff stress tensor can be obtained by

$$
\boldsymbol{S}=\frac{\partial \Psi(\boldsymbol{E})}{\partial \boldsymbol{E}}=\mathbf{C}: \boldsymbol{E}
$$

in which $\mathbf{C}$ is the fourth-order constitutive (elastic) tensor.

Although it is well known that the Kirchhoff-Saint Venant model is unstable under compression, and thus of limited importance in engineering applications, we have considered it here for the sake of simplicity in the formulation and because it is still in use in fields such as real-time simulation of surgery and rendering in general, where it is among the state of the art models [35] [33] [7].

Under these assumptions, the ANM is based upon expanding the displacement in the neighborhood of each material point in terms of a control parameter " $a$ ". This expansion is developed in the neighborhood of a known equilibrium point $\left(\boldsymbol{u}^{m} ; \boldsymbol{S}^{m} ; \bar{\lambda}^{m}\right)$ at step $m$ and the series is truncated at order $N$. To simplify the resulting expressions, also the second Piola-Kirchhoff stress tensor and the load parameter $\lambda$ are expanded in series prior to their introduction in the weak form of the problem:

$$
\left\{\begin{array}{l}
\boldsymbol{u}^{m+1}(a) \\
\boldsymbol{S}^{m+1}(a) \\
\bar{\lambda}^{m+1}(a)
\end{array}\right\}=\left\{\begin{array}{l}
\boldsymbol{u}^{m}(a) \\
\boldsymbol{S}^{m}(a) \\
\bar{\lambda}^{m}(a)
\end{array}\right\}+\sum_{p=1}^{N} a^{p}\left\{\begin{array}{c}
\boldsymbol{u}_{p} \\
\boldsymbol{S}_{p} \\
\bar{\lambda}_{p}
\end{array}\right\}
$$

where $\left(\boldsymbol{u}_{p}, \boldsymbol{S}_{p}, \bar{\lambda}_{p}\right)$ are unknowns. Above, $\left(\boldsymbol{u}^{m+1}(a), \boldsymbol{S}^{m+1}(a), \bar{\lambda}^{m+1}(a)\right)$ represents the solution along a portion of the loading curve. Noteworthy, the behavior of the solid is described continuously with respect to " $a$ ". The introduction of Eq. (26) into Eq. (21) and Eq. (25) leads to a series of linear problems with the same tangent operator, thus avoiding the burden associated with stiffness matrix updating in the Newton-Raphson scheme.

In general, any variable can be expanded in terms of $a$, so, for instance, the series expansion of $\boldsymbol{E}^{*}(\boldsymbol{u})$ gives

$$
\boldsymbol{E}^{*^{m+1}}(a)=\gamma_{l}\left(\boldsymbol{u}^{*}\right)+\gamma_{n l_{S}}\left(\boldsymbol{u}^{*}, \boldsymbol{u}^{m}\right)+\sum_{p=1}^{N} a^{p} \gamma_{n l_{S}}\left(\boldsymbol{u}^{*}, \boldsymbol{u}_{p}\right)
$$

As can be noticed, $\boldsymbol{E}^{*^{m+1}}$ includes terms in both $\boldsymbol{u}$ and $\boldsymbol{u}^{*}$. This is not surprising, since we are computing a correction to $\boldsymbol{u}^{m}$. The series expansions of $\boldsymbol{S}$ gives in turn

$$
\begin{aligned}
& \boldsymbol{S}^{m+1}(a)=\mathbf{C}: \boldsymbol{E}^{m+1}(a)= \\
& \quad \mathbf{C}:\left[\gamma_{n l}\left(\boldsymbol{u}^{m}, \boldsymbol{u}^{m}\right)+\gamma_{l}\left(\boldsymbol{u}^{m}\right)+\sum_{p=1}^{N} a^{p}\left(\gamma_{l}\left(\boldsymbol{u}_{p}\right)+\gamma_{n l_{S}}\left(\boldsymbol{u}^{m}, \boldsymbol{u}_{p}\right)+\sum_{i=1}^{p-1} \gamma_{n l}\left(\boldsymbol{u}_{i}, \boldsymbol{u}_{p-i}\right)\right)\right],
\end{aligned}
$$

and at order $p$ we obtain

$$
\boldsymbol{S}_{p}=\mathbf{C}:\left\{\gamma_{l}\left(\boldsymbol{u}_{p}\right)+\gamma_{n l_{S}}\left(\boldsymbol{u}^{m}, \boldsymbol{u}_{p}\right)+\sum_{i=1}^{p-1} \gamma_{n l}\left(\boldsymbol{u}_{i}, \boldsymbol{u}_{p-i}\right)\right\}
$$

Introducing the asymptotic expansion into Eq. (25) results in

$$
\begin{array}{r}
\int_{\Omega_{0}}\left\{\left(\boldsymbol{S}^{m}+\sum_{p=1}^{N} a^{p} \boldsymbol{S}_{p}\right):\left(\gamma_{l}\left(\boldsymbol{u}^{*}\right)+\gamma_{n l_{S}}\left(\boldsymbol{u}^{m}, \boldsymbol{u}^{*}\right)+\sum_{p=1}^{N} a^{p} \gamma_{n l_{S}}\left(\boldsymbol{u}_{p}, \boldsymbol{u}^{*}\right)\right)\right\} d \Omega= \\
\left(\bar{\lambda}^{m}+\sum_{p=1}^{N} a^{p} \bar{\lambda}_{p}\right) \Psi_{e x t}\left(\boldsymbol{u}^{*}\right),
\end{array}
$$


with $\boldsymbol{\Psi}_{\text {ext }}\left(\boldsymbol{u}^{*}\right)=\int_{\Gamma_{t}} \boldsymbol{t} \cdot \boldsymbol{u}^{*} d \Gamma$. Introducing Eq. (29) into Eq. (30) and identifying terms with the same power of " $a$ " results in a successive series of linear problems which at order $p,(p=1, \ldots, N)$ takes the form

$$
\mathcal{L}\left(\boldsymbol{u}^{*}, \boldsymbol{u}^{m+1}\right)=\bar{\lambda}_{p} \boldsymbol{\Psi}_{e x t}\left(\boldsymbol{u}^{*}\right)+F_{p}^{n l}\left(\boldsymbol{u}^{*}\right)
$$

with

$\mathcal{L}\left(\boldsymbol{u}^{*}, \boldsymbol{u}^{m+\mathbf{1}}\right)=\int_{\Omega}\left\{\boldsymbol{S}^{m}: \gamma_{n l_{S}}\left(\boldsymbol{u}^{m}, \boldsymbol{u}^{*}\right)+\left[\gamma_{l}\left(\boldsymbol{u}^{*}\right)+\gamma_{n l_{S}}\left(\boldsymbol{u}_{p}, \boldsymbol{u}^{*}\right)\right]: \mathbf{C}:\left[\gamma_{l}\left(\boldsymbol{u}_{p}\right)+\gamma_{n l_{S}}\left(\boldsymbol{u}^{m}, \boldsymbol{u}_{p}\right)\right]\right\} d \Omega$

and where $\boldsymbol{F}_{p}^{n l}\left(\boldsymbol{u}^{*}\right)$ is equal to zero at order one and at order $p$ it can be calculated as

$$
F_{p}^{n l}\left(\boldsymbol{u}^{*}\right)=-\int_{\Omega}\left\{\sum_{i=1}^{p-1} \boldsymbol{S}_{i}: \gamma_{n l_{S}}\left(\boldsymbol{u}_{p-i}, \boldsymbol{u}^{*}\right)+\sum_{i=1}^{p-1}\left[\gamma_{n l}\left(\boldsymbol{u}_{i}, \boldsymbol{u}_{p-i}\right)\right]: \mathbf{C}:\left[\gamma_{l}\left(\boldsymbol{u}^{*}\right)+\gamma_{n l_{S}}\left(\boldsymbol{u}^{m}, \boldsymbol{u}^{*}\right)\right]\right\} d \Omega
$$

Discretization of Eq. (31) by using finite elements leads to a sequence of linear problems in the form [17]

$$
\begin{gathered}
\text { Order } 1\left\{\begin{array}{c}
\boldsymbol{K}_{t} \boldsymbol{u}_{1}=\bar{\lambda}_{1} \boldsymbol{f} \\
\boldsymbol{u}_{1}^{T} \boldsymbol{u}_{1}+\bar{\lambda}_{1}^{2}=1
\end{array}\right. \\
\text { Order } p\left\{\begin{array}{l}
\boldsymbol{K}_{t} \boldsymbol{u}_{p}=\bar{\lambda}_{p} \boldsymbol{f}+\boldsymbol{f}_{p}^{n l}\left(\boldsymbol{u}_{i}\right) \quad i<p \\
\boldsymbol{u}_{p}^{T} \boldsymbol{u}_{1}+\bar{\lambda}_{p} \lambda_{1}=0
\end{array}\right.
\end{gathered}
$$

where $\boldsymbol{K}_{t}$ denotes the tangent stiffness matrix associated with Eq. (32), common to the problems at different orders $p$. It is the same as the one applied in a classical iterative algorithm like NewtonRaphson (in the first iteration). In the above, $\boldsymbol{u}_{p}$ is the discretized form of the displacement field at order $p, \boldsymbol{f}$ is the loading vector and $\boldsymbol{f}_{p}^{n l}$ represents the discretized form associated with $\boldsymbol{F}_{p}^{n l}\left(\boldsymbol{u}^{*}\right)$ in Eq. (33), which at order $p$ only depends on the values of $\boldsymbol{u}_{i}, i<p$.

\section{A combined PGD-ANM approach to hyperelasticity}

The combination of the two previously introduced tools, namely the PGD approach to parametrized problems (in this case the position of the load is the parameter) and the ANM for a consistent linearization of the weak form of the problem gives rise to a particularly useful formulation. In it, the displacement field is approximated as a series expansion around the last equilibrium point, whereas each term of the series is considered to be approximated by a finite sum of separated functions:

$$
\boldsymbol{u}^{m+1}=\boldsymbol{u}^{m}+a \sum_{i=1}^{n_{1}}\left(\boldsymbol{F}_{i}^{1} \circ \boldsymbol{G}_{i}^{1}\right)+a^{2} \sum_{j=1}^{n_{2}}\left(\boldsymbol{F}_{j}^{2} \circ \boldsymbol{G}_{j}^{2}\right)+\ldots+a^{p} \sum_{l=1}^{n_{p}}\left(\boldsymbol{F}_{l}^{p} \circ \boldsymbol{G}_{l}^{p}\right)
$$

This gives rise to a series of problems of the form (34)-(35) within which a traditional PGD problem is solved for functions $\boldsymbol{F}_{i}^{j}(\boldsymbol{x})$ and $\boldsymbol{G}_{i}^{j}(\boldsymbol{s})$ at each order of the expansion, $j$. No further modification of the method is necessary, resulting in a series of standard PGD problems that can be solved by employing any of the available non-linear solvers. Here, as in many of our previous works, we have employed a fixed point algorithm, similar to the one sketched in Sections 2.1-2.2 before.

We study the behavior of the proposed technique by means of a series of benchmark problems in Section 5. 


\section{Numerical examples}

\subsection{Kirchhoff-Saint Venant material}

We will consider two sets of examples. The first one is composed by three different benchmark tests over Kirchhoff-Saint Venant materials. Despite its simplicity and well-known limitations, KirchhoffSaint Venant approaches are very useful in the field of real-time simulation, since they provide a good compromise between realism in the deformation and computational cost [44] [7].

5.1.1. Pinched cube A unit cube modeled by $3 \times 3 \times 3$ nodes and a tetrahedral mesh is considered. Young's modulus of $1 \mathrm{MPa}$ and Poisson's coefficient of 0.25 are assumed. The cube is loaded by a vertical force $(0.01 \mathrm{~N})$ acting at any point of the top face. Results obtained with the before presented PGD-ANM approach are compared to traditional FE approaches, solved by standard Newton-Raphson linearization strategies. For one particular position of the load (one corner) the load-displacement curve is shown in Fig. 1, while its deformed configuration is shown in Fig. 2.

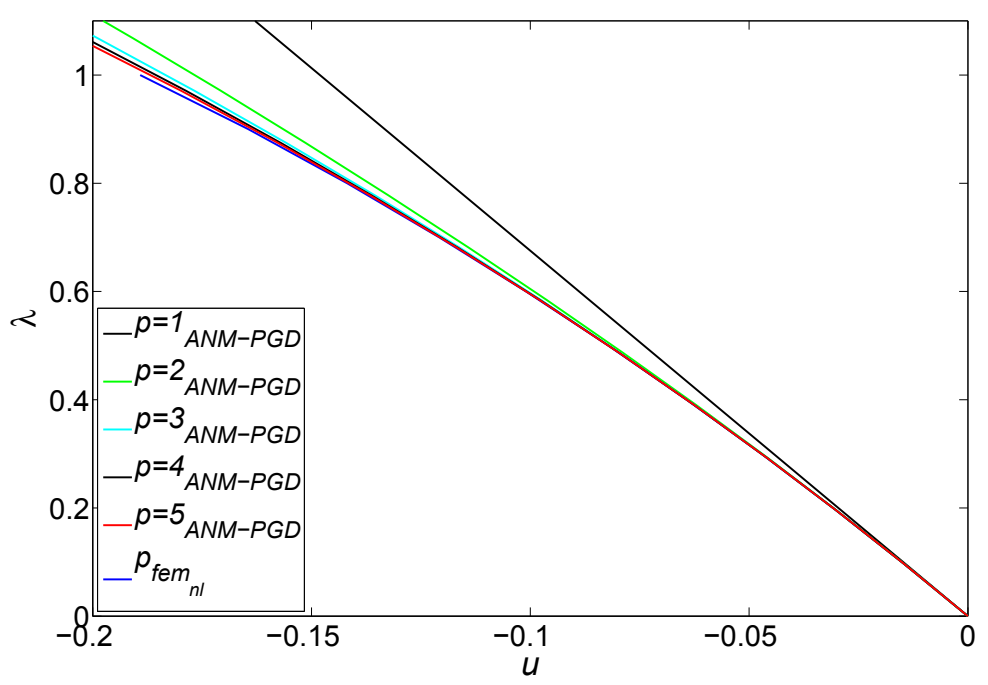

Figure 1. Load-displacement curve (in terms of the load parameter $\lambda$ ) for one particular load position of the pinched cube problem. Different solutions for different orders of expansion $(p=1, \ldots, 5)$ compared to the FE solution by employing Newton-Raphson algorithms.

In general, as was the case in previous references such as [35], expansions up to order 6 are enough to obtain a reasonable accuracy, despite the fact that in the ANM community much higher order expansions are usually employed, on the order of 15 [16]. The number of modes (separated functions) employed at each expansion term in this example was 17, 17, 11, 2, 1 and 1, respectively, for therms of order 1 to 6 . The accuracy of the approach is noteworthy, despite the fact that terms 5 and 6 of the expansion (solution for the $6^{\text {th }}$-order expansion is not depicted in Fig. 1, because it is practically indistinguishable from that of order 5) are obtained with only one couple $\boldsymbol{F}(\boldsymbol{x}) \circ \boldsymbol{G}(\boldsymbol{s})$, i.e., $n_{5}=n_{6}=1$ in Eq. (36). 


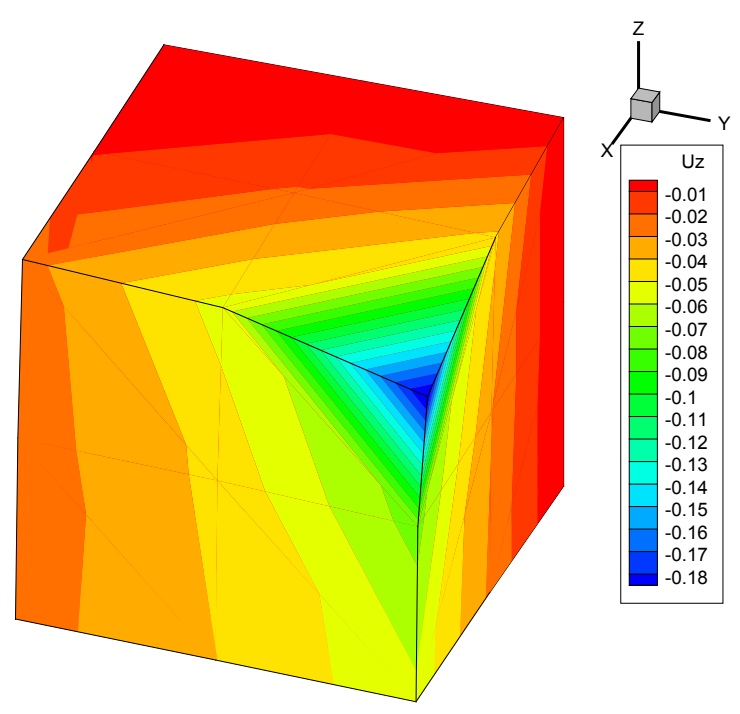

Figure 2. Deformed configuration of the cube for one particular load position.

5.1.2. Beam bending In this case we consider the problem of beam bending. This simple test is among the most popular ones in the field of real-time simulation, since it readily shows a great divergence from physical results if a poor formulation is used [2]. In fact, if linear elasticity formulations are employed, a great gain of volume is observed, leading the observer to perceive a clearly non-physical result, see Fig. 3.

In this case we consider a squared cross-section beam, with $40 \times 40 \times 400 \mathrm{~mm}$. Young's modulus was assumed to be $209000 \mathrm{MPa}$, while Poisson coefficient was set to 0.3 . A load of $10^{6} \mathrm{~N}$ can be applied at any point of the upper face of the beam. Under these conditions, a comparison was established between the tip displacement obtained for the load position at the beam rightmost side with the value obtained using standard FE analysis and Newton-Raphson iterations to solve the non-linear equations.

Results for different expansion order are shown in Fig. 4. For a $5^{\text {th }}$-order expansion, almost no difference can be perceived between the FE result and the reduced PGD-ANM result. For this example, the number of separated functions necessary at each expansion order was 162, 102, 79, 161, 24, and 8, respectively. As stopping criterion, we employed a relative one, which stops the fixed point algorithm if the new functional pair contributes less than $10^{-4}$ times the initial pair of functions, and a general one in which the algorithm is stopped if the modulus of the new functional pair is less than $10^{-15}$.

In general, results are below $5 \%$ error with respect to the target FE solution of the problem. In any case, a higher number of modes for each term, or a higher number of terms in the expansion, has been shown to converge to the right solution. It is true, in general, that the number of off-line computed modes depends on the error estimator considered (See the works by Huerta [3], or Chamoin and Ladeveze [27]). At present there are not efficient and robust error estimators applicable to general multi-parametric non-linear models. For this reason we computed many terms (remember that this calculation is carried out off-line and only once) and then we compared the solution for some particular choices of the parameters with the one obtained with finite elements in order to check the convergence 


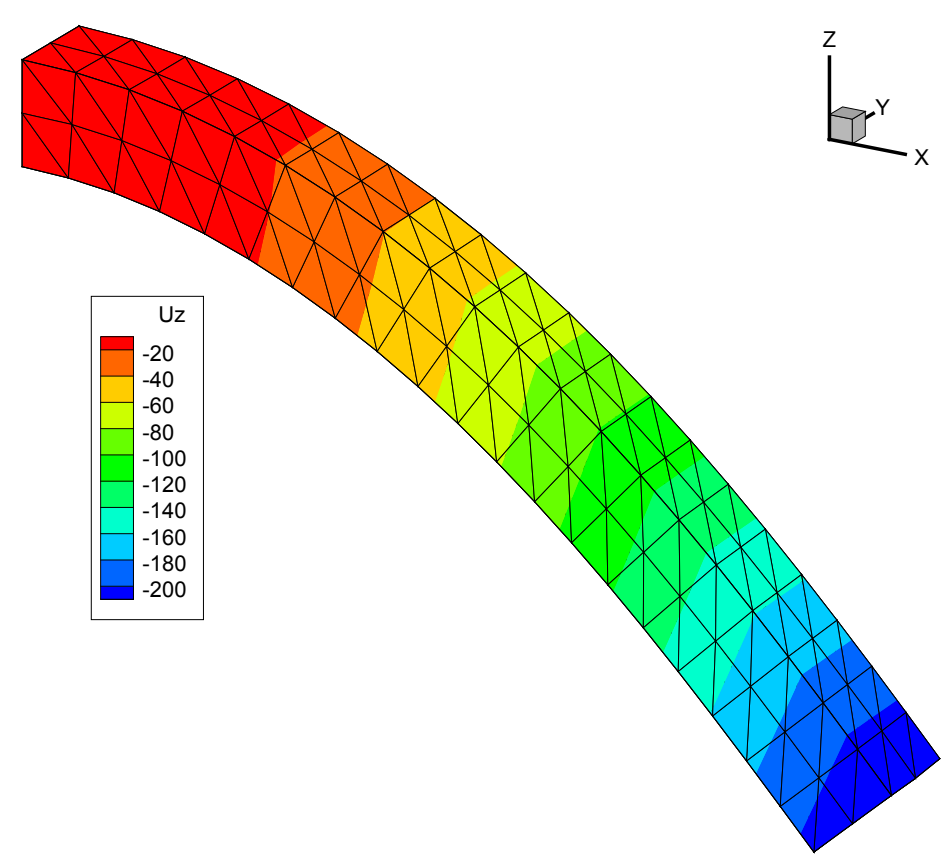

Figure 3. Deformed configuration of the beam for the load positioned at the beam tip. Note that no artificial gain in volume can be perceived.

and solution accuracy.

5.1.3. Palpation of the liver One of the most typical examples in real-time applications is that of liver palpation in a laparoscopic virtual surgery procedure. The liver is the biggest gland in the human body, after the skin. Liver geometry has been obtained from the SOFA project [2] and post-processed in order to obtain a mesh composed by 2853 nodes and 10519 tetrahedra, whose geometry is shown in Fig. 5. The anterior surface of the liver is considered free, while the posterior one was assumed to be supported over different organs (it is connected to the diaphragm by the coronary ligament, for instance). The inferior vena cava travels along the posterior surface, and the liver is frequently assumed clamped a that location. Although the assumed boundary conditions are not strictly correct from a physiological point of view, our main interest is to show that the model can be solved under real-time constraints with reasonable accuracy.

Here, the human liver is considered as a Kirchhoff-Saint Venant material with $E=0.17 \mathrm{MPa}$ and $\nu=0.48$ [18]. A vertical load of $5 \mathrm{~N}$ is considered at any point of the anterior surface of the liver. Again, for comparison purposes, results are evaluated at a particular position of the load and compared with those obtained with a traditional FE analysis and standard Newton-Raphson iterative schemes. It can be noticed how the $6^{\text {th }}$-order expansion gives very reasonable results if compared to that of the FE model.

The vertical displacement field for that particular position of the load is depicted in Fig. 7. To check the overall behavior of the technique, functions $\boldsymbol{F}_{i}$ and $\boldsymbol{G}_{i}$ (see Eq. (36)) for $i=1,2,3$ and 20 (terms of first and fourth-order expansions) are shown in Figs. 8-11. 


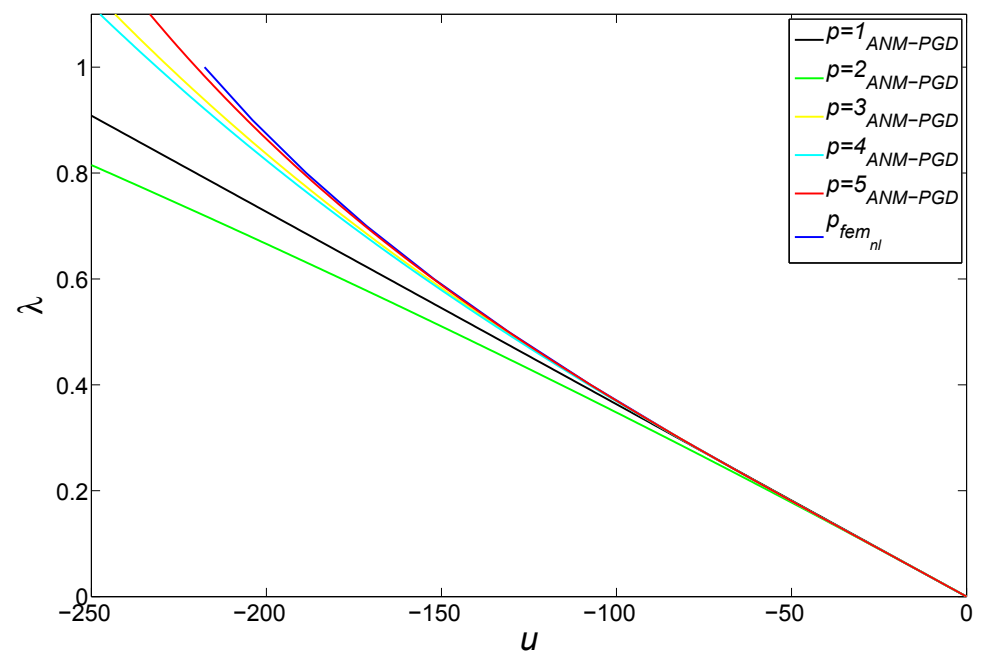

Figure 4. Load-displacement curve (in terms of the load parameter $\lambda$ ) for one particular load position of the beam bending problem. Different solutions for different orders of expansion $(p=1, \ldots, 5)$ compared to the FE solution by employing Newton-Raphson algorithms.
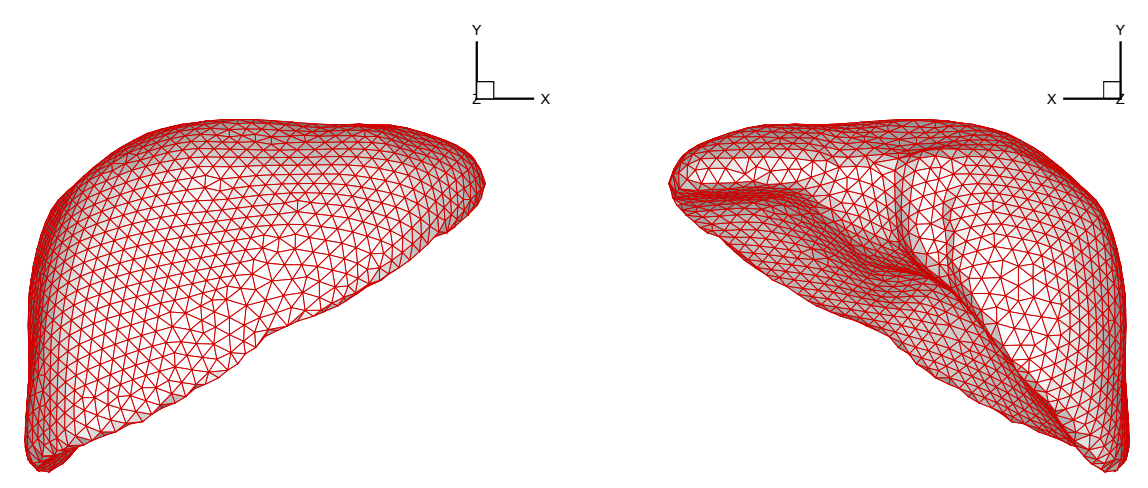

Figure 5. Geometry of the finite element model for the liver.

\subsection{Neo-hookean behavior}

Extension of the before presented technique to neo-hookean materials [10] is in principle straightforward, although lengthy (this is not important, in fact, since the parametric calculation is done off-line). The major difference with the Kirchhoff-Saint Venant model is the presence of material non-linearities, in addition to the geometrical ones. A POD-ANM approach has been suggested in a previous work of the authors, see [35], and is here extended to the before presented PGD framework. 


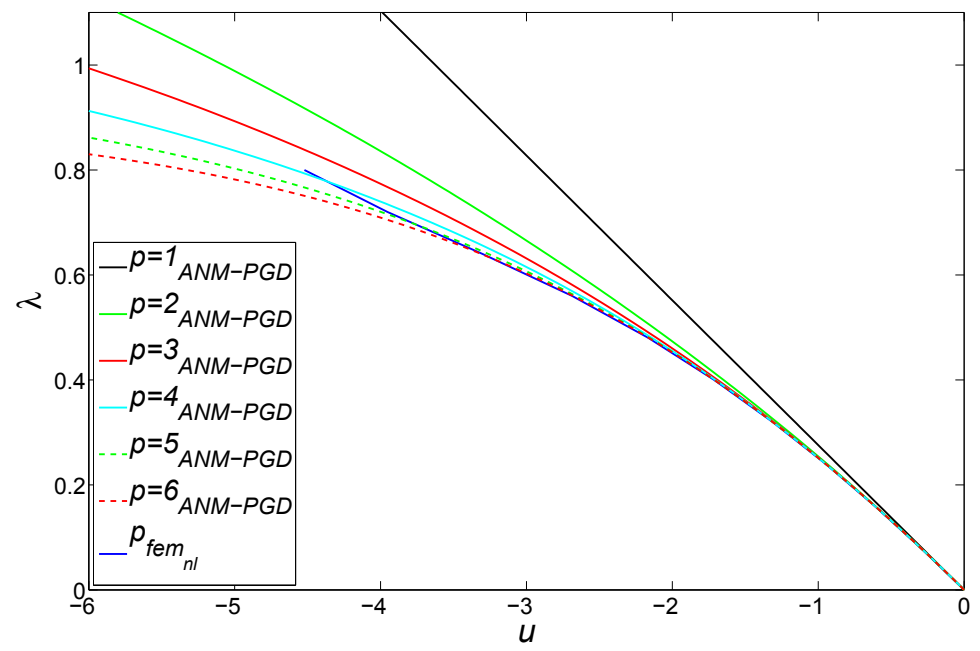

Figure 6. Load-displacement curve (in terms of the load parameter $\lambda$ ) for one particular load position of the liver palpation problem. Different solutions for different orders of expansion $(p=1, \ldots, 6)$ compared to the FE solution by employing Newton-Raphson algorithms. Noticeably, the Newton-Raphson algorithm did not converge when solving the case $\lambda=1$.

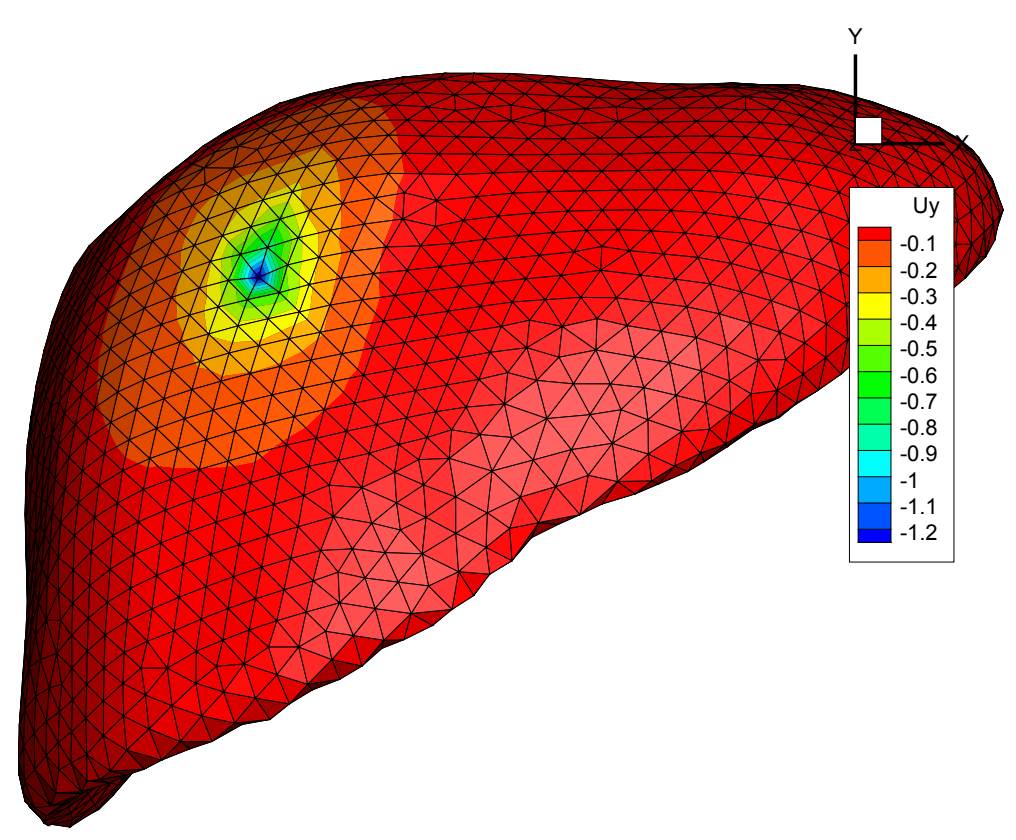

Figure 7. Vertical displacement field of the Kirchhoff-Saint Venant liver for one particular position of the load. 


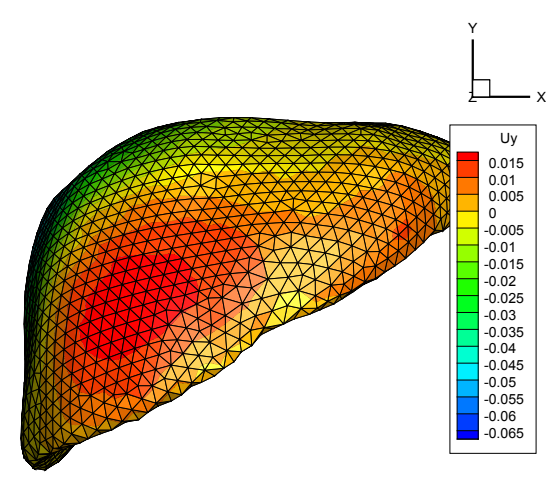

(a)

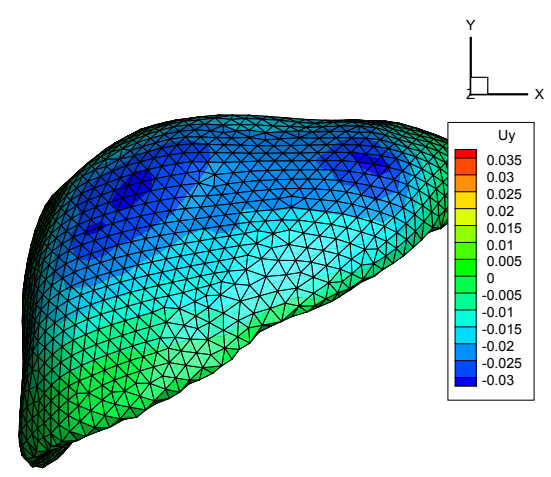

(c)

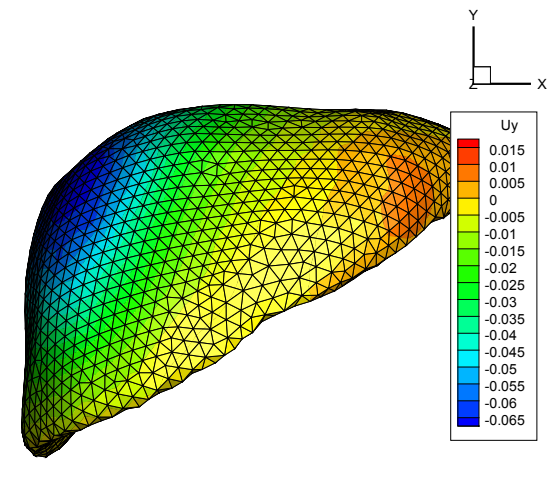

(b)

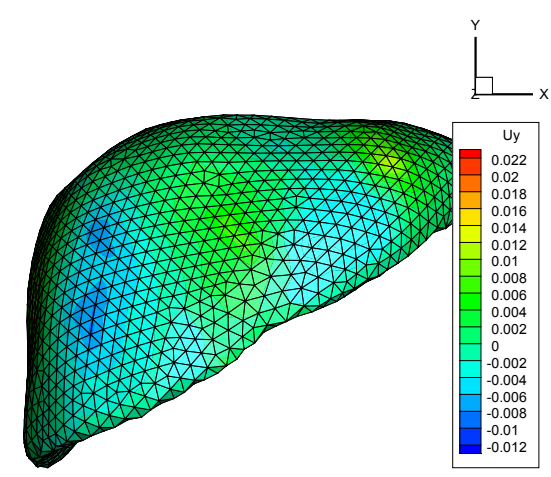

(d)

Figure 8. Functions $\boldsymbol{F}_{i}^{1}(\boldsymbol{x})$, for $i=1,2,3$ and 20, respectively, for the first-order expansion, in the simulation of the Kirchhoff-Saint Venant liver. 


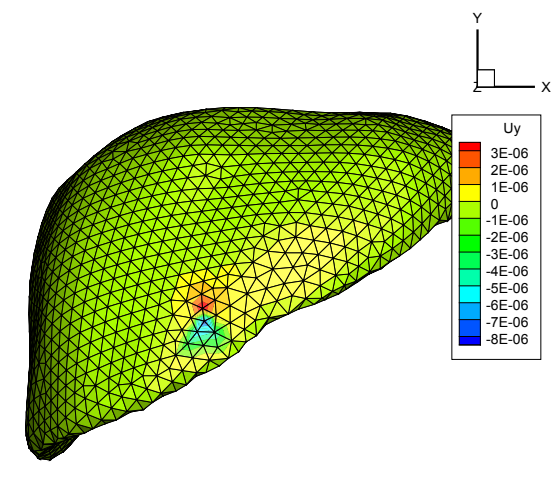

(a)

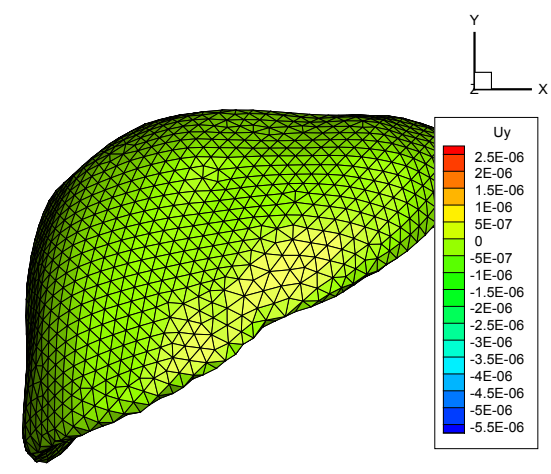

(c)

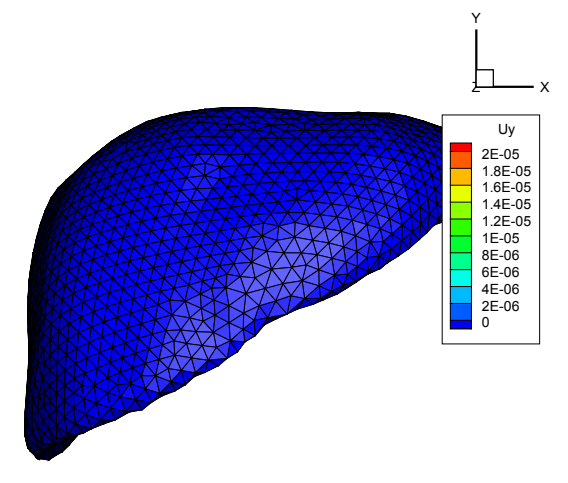

(b)

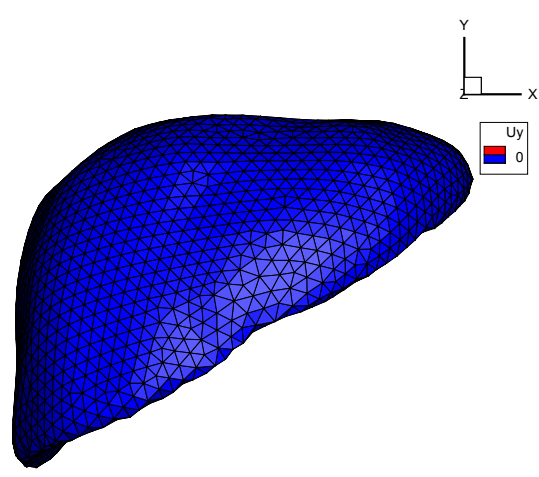

(d)

Figure 9. Functions $\boldsymbol{F}_{i}^{4}(\boldsymbol{x})$, for $i=1,2,3$ and 20, respectively, for the fourth-order expansion, in the simulation of the Kirchhoff-Saint Venant liver. 


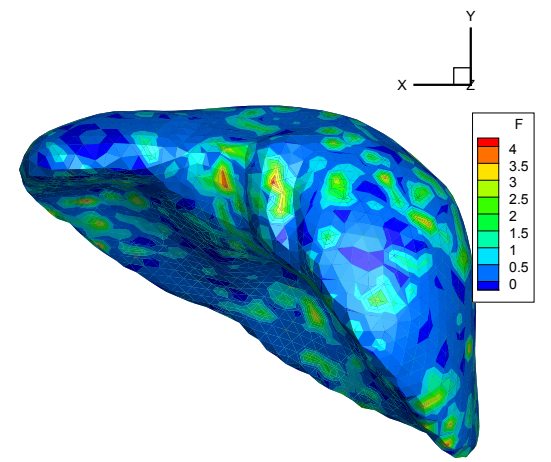

(a)

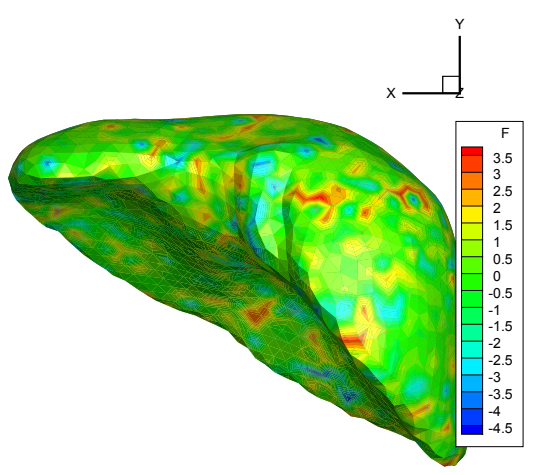

(c)

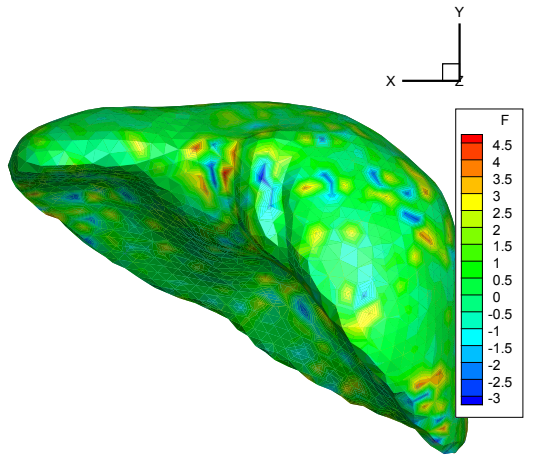

(b)

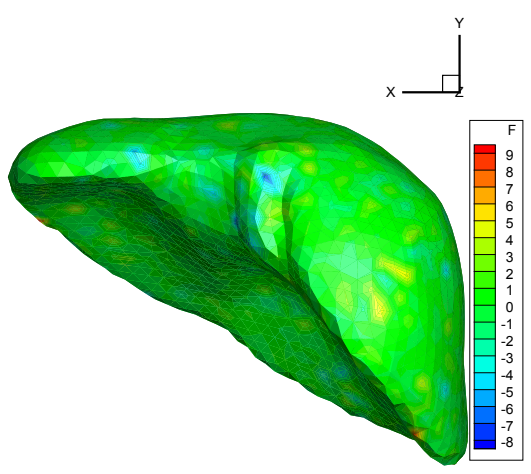

(d)

Figure 10. Functions $G_{i}^{1}(\boldsymbol{s})$, for $i=1,2,3$ and 20, respectively, for the first-order expansion, in the simulation of the Kirchhoff-Saint Venant liver. 


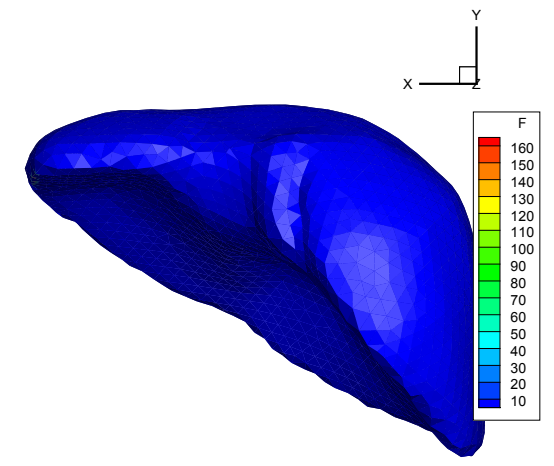

(a)

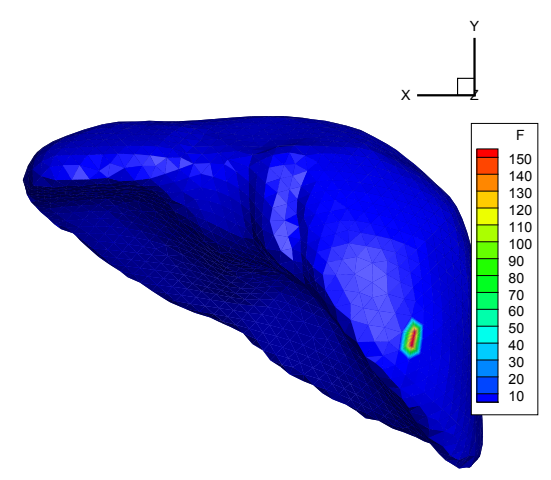

(c)

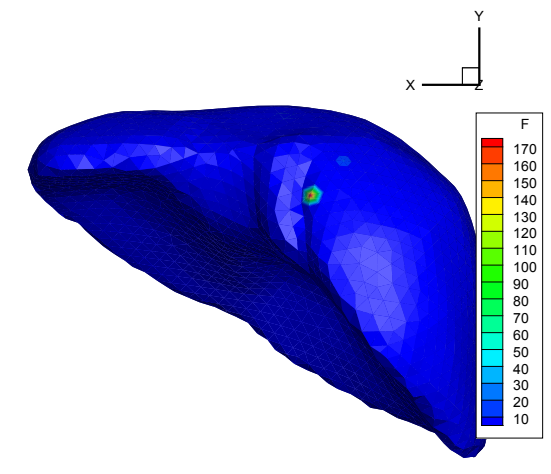

(b)

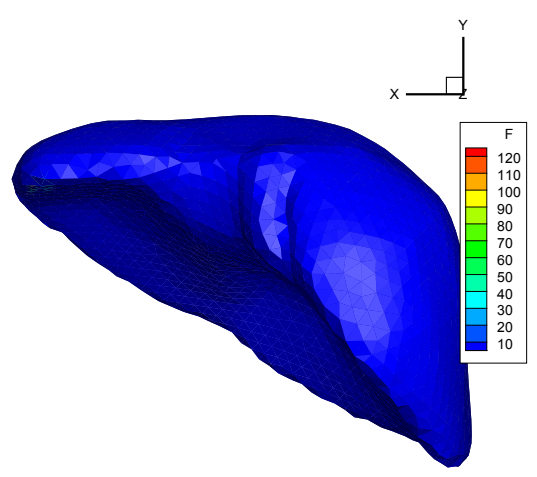

(d)

Figure 11. Functions $G_{i}^{4}(\boldsymbol{s})$, for $i=1,2,3$ and 20, respectively, for the fourth-order expansion, in the simulation of the Kirchhoff-Saint Venant liver. 
The compressible Neo-Hookean model is characterized by a strain energy function given by

$$
\Psi=\frac{\mu}{2}(\operatorname{tr}(\boldsymbol{C})-3)-\mu \ln J+\frac{\lambda}{2}(\ln J)^{2}
$$

where $\lambda$ and $\mu$ are Lame's constants and $\boldsymbol{C}=\boldsymbol{I}+2 \boldsymbol{E}$ is the right Cauchy-Green strain tensor. The second Piola-Kirchhoff stress tensor can be obtained by

$$
\boldsymbol{S}=\frac{\partial \Psi(\boldsymbol{E})}{\partial \boldsymbol{E}}=\mu\left(\boldsymbol{I}-\boldsymbol{C}^{-1}\right)+\lambda(\ln J) \boldsymbol{C}^{-1} .
$$

In this case the intricate expansion procedure becomes easier if we identify, as in [12], the asymptotic expansion with a Taylor series of the variables of interest, denoted by $\boldsymbol{U}(a)$, in the vicinity of $a=0$. Truncating at $\operatorname{order} N$ :

$$
\boldsymbol{U}(a)=\boldsymbol{U}_{0}+\sum_{p=1}^{N} \boldsymbol{U}_{p} a^{p}
$$

where $\boldsymbol{U}_{0}=\boldsymbol{U}(0)$ and

$$
\left.\boldsymbol{U}_{p}=\frac{1}{p !} \frac{d^{p} \boldsymbol{U}}{d a^{p}}\right\rfloor_{a=0} .
$$

In this case, as in [33], we have selected the following variables to perform the expansion:

$$
\boldsymbol{U}(a)=\left(\begin{array}{c}
\boldsymbol{u}(a) \\
\boldsymbol{S}(a) \\
J^{2} \boldsymbol{C}^{-1}(a) \\
\ln \sqrt{J^{2}}(a) \\
\frac{1}{J^{2}}(a) \\
\lambda(a)
\end{array}\right)
$$

By performing the substitution of the before mentioned variables into the weak form of the problem, see Eq. (21) we arrive to a problem entirely similar to that in Eqs. (34) and (35). The entire details are provided, for completeness, in Appendix 1.

5.2.1. Neo Hookean beam under bending We reproduce here the problem in Section 5.1.2 but considering a neo Hookean constitutive model. The deformed configuration of the beam at expansion orders 1 to 4 is depicted in Fig. 12. Note how the first-order expansion (linear approach) shows a tremendous gain in volume that renders the simulations clearly non-physical. Again, expansions up to order 4 to 6 were judged sufficient to obtain a good approximation to the reference solution, see Fig. 13. Obviously, higher accuracy can be obtained by increasing even more the expansion order. Up to $p=15$ is a typical value of the expansion order in the ANM literature.

5.2.2. Palpation of a neo-hookean liver The same procedure has been applied to the problem in Section 5.1.3 but now considering neo-hookean behavior. The neo-hookean law in Eq. (37) has been now particularized to $E=0.17 \mathrm{MPa}$ and $\nu=0.48$. As in previous examples, a PGD-ANM solution has been obtained and compared to a standard finite element solution at a particular node. To this end, standard Newton-Raphson procedures have been employed for the solution of the resulting non-linear system of equations. The load-displacement curve for this particular node is shown in Fig. 14. 


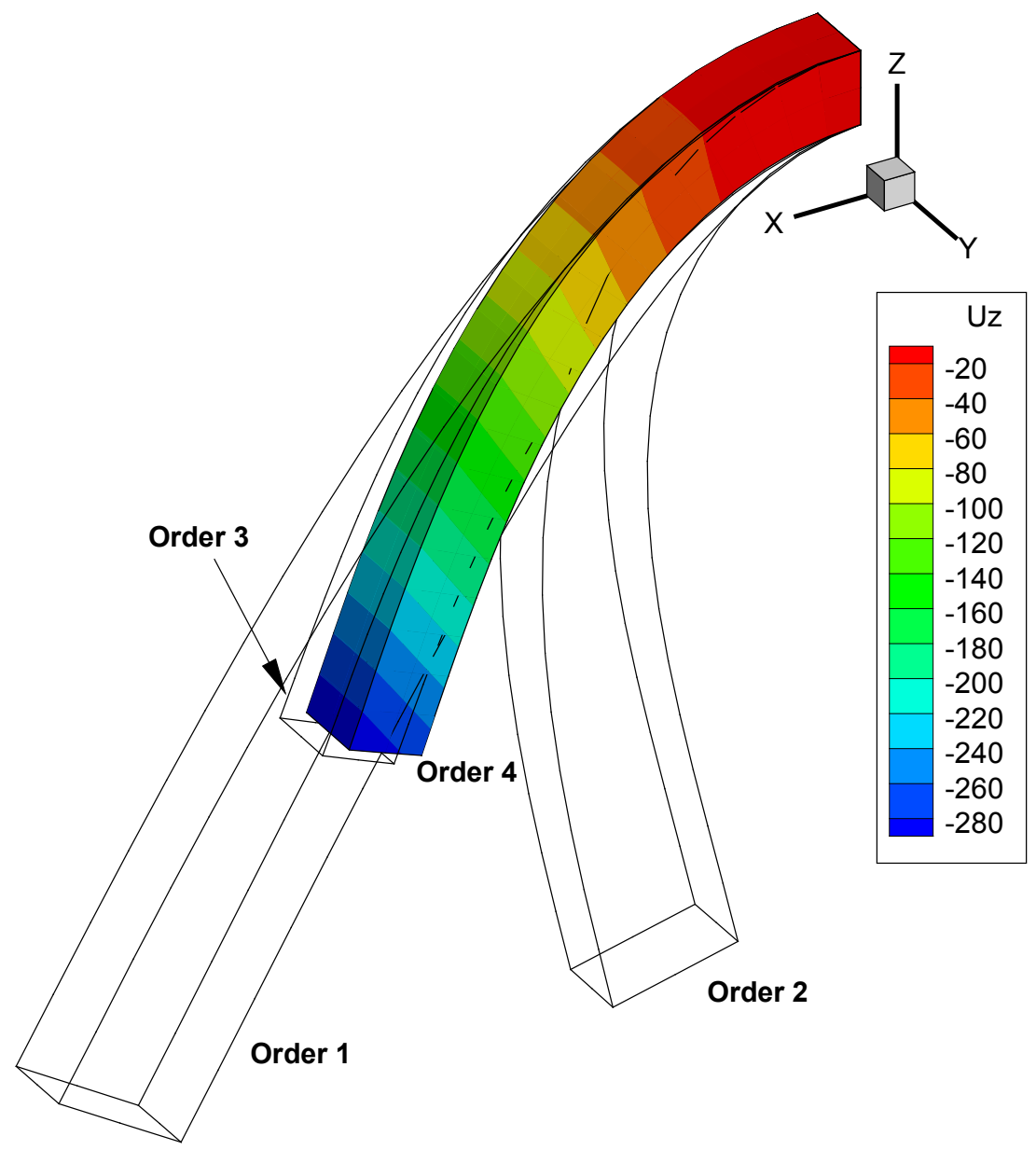

Figure 12. Solution for the neo-hookean beam bending problem at different expansion orders.

In this case the observed agreement between the PGD-ANM solution and that of the finite element model is even higher than in the previous example. For a fourth order expansion, the agreement between the predicted load-displacement curves is almost exact.

Remember that our approach is based upon an off-line/on-line procedure such that, once the off-line computation has been done, its solution is stored in the form of a series of one-dimensional vectors that are evaluated in real time very efficiently. The solution thus computed is a multidimensional one, that is particularized on-line. This procedure is sketched in Fig. 15. 


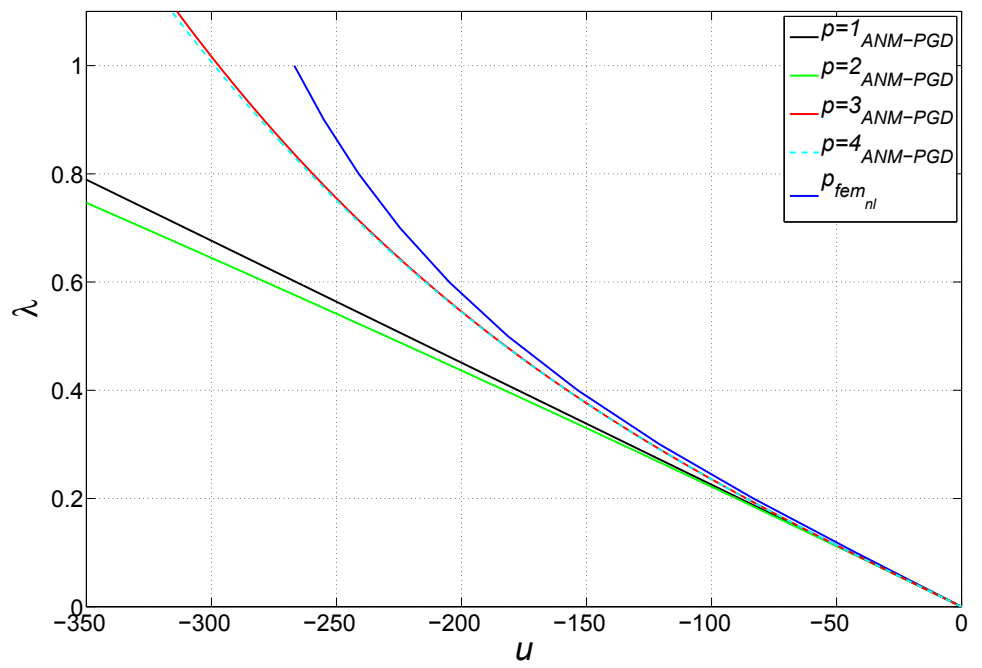

Figure 13. Solution for the neo-hookean beam bending problem at different expansion orders.

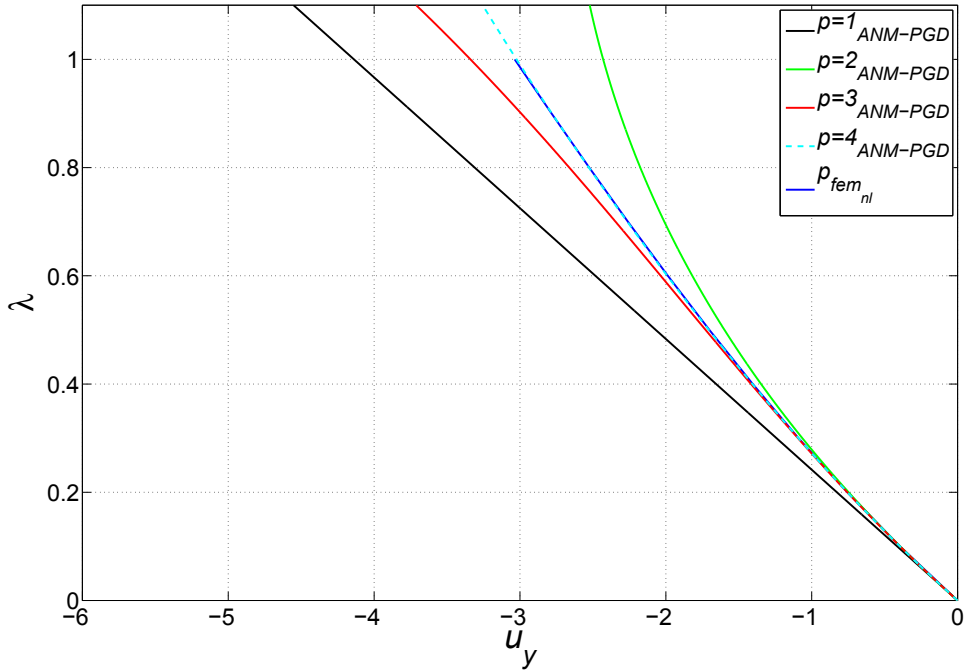

Figure 14. Comparison between the PGD-ANM solution, at different expansion orders, and that for a standard finite element solution for a load at a particularized position. Neo-Hookean behavior. 


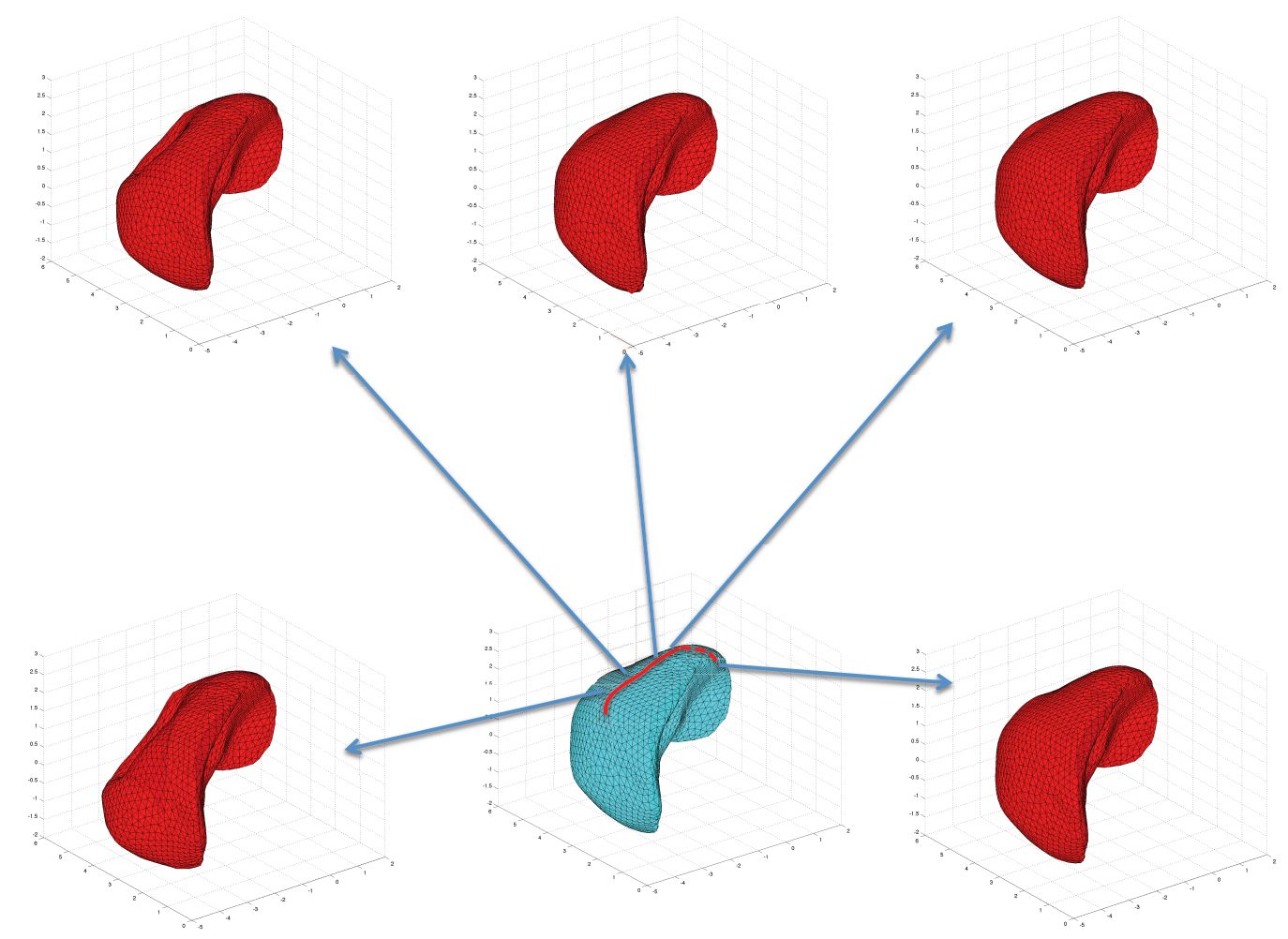

Figure 15. The result of the off-line PGD simulation is a multidimensional solution that depends on the load position. Thus, evaluating the solution for a prescribed load is entirely similar to performing a cut on a hyper volume $\Omega \times \bar{\Gamma}$. In the figure, the red line on the blue liver represents approximately the path followed by the load over the surface of the liver. The displacement is magnified by a factor of 2.0.

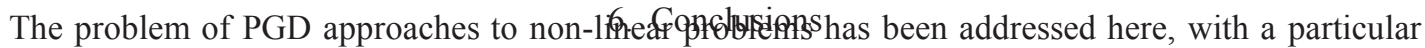
emphasis on hyperelasticity. The development of suitable linearizations for complex non-linear problems formulated under a PGD framework has been a major focus of attention for our community in the last years. Firstly because consistent linearizations of the complex weak forms appearing in these formulations are far from being readily available. Previous works include explicit approaches to these problems [34] but are difficult to generalize to arbitrary hyperelastic models or constitutive laws. The presented technique could be applied to any hyperelastic constitutive law, and, in view of existing results in the ANM, possibly to other non-linearities such as plasticity.

Here we have proposed a combined PGD-Asymptotic Numerical Method approach. The main advantages of this approach is that it produces a series approach to the solution that involves the same tangent operator for all the terms in the expansion. Thus costly stiffness matrix updating procedures, that constitute nowadays one of the main problems in the model order reduction community, are 
avoided. It has been shown how the proposed method provides with a very accurate solution to complex problems in the form of a meta-model that can be evaluated at very high feedback rates (even at real time rates on the order of $1 \mathrm{kHz}$ ) with very little computational cost. This is precisely one of the most attractive features of the just proposed method. It combines and off-line/on-line procedure by which a truncated expansion of the multi-dimensional form of the displacement is obtained and stored in memory. After, these modes are evaluated in the on-line procedure of the method at extremely fast feedback rates. We believe that this philosophy opens new possibilities in the field of real-time simulation that deserve to be explored.

Other problems remain open, however. Notably, optimality of PGD approaches (i.e., under what circumstances a priori PGD modes are equivalent to a posteriori POD or SVD modes) is not well understood. It has been noticed in the examples throughout this work that, unlike those in previous explicit approaches, PGD-ANM modes seem not to be optimal. They are highly oscillating and numerous for a prescribed tolerance. The solution is simple, however. It suffices to obtain the SVD modes that show most of the energy of the system to get a very compact representation of the solution (the so-called projected PGD, see [36]). But in any case, it seems pertinent to work in discerning what are the key ingredients of optimality in a PGD approach. This constitutes one of our current efforts of research.

In any case, although not optimal, the proposed method provides with a very competitive solution to highly demanding problems in applied sciences and engineering such as dynamic data-driven problems, real-time response even under very restrictive scenarios (haptic peripherals, for instance), simulation-driven control of structures and processes, and many others, where non-linear simulations are nowadays standard in industrial practice.

\section{APPENDIX}

I. Derivation of the tangent stiffness matrix for the neo-hookean case

For the neo-hookean case explained in Section 5.2, the tangent stiffness matrix takes the following form:

$$
\boldsymbol{K}_{t}=\int_{\Omega_{0}}\left(\boldsymbol{B}^{T} \boldsymbol{D} \boldsymbol{B}+\boldsymbol{G}^{T} \tilde{\boldsymbol{S}_{0}} \boldsymbol{G}\right) d \Omega
$$

where

$$
\boldsymbol{D}=\lambda\left(\frac{1}{J_{0}^{2}} \boldsymbol{C}_{0}^{-1} \boldsymbol{M}_{0}^{T}\right)+2\left(\mu-\lambda \ln J_{0}\right)\left(\frac{1}{J_{0}^{2}}\left(\boldsymbol{C}_{0}^{-1} \boldsymbol{M}_{0}^{T}\right)-\tilde{\boldsymbol{C}}_{0}\right)
$$

now takes into account the material non-linearity and has a somewhat similar appearance to the Lagrangian elastic tensor at the initial state. $J_{0}$ and $\boldsymbol{C}_{0}$ represent the Jacobian and right CauchyGreen strain tensor of the initial solution. $M_{0}$ is obtained from the series expansion of the Jacobian, and contains minors of $\boldsymbol{C}_{0}$. Finally, $\tilde{\boldsymbol{C}}_{0}$ is obtained from the series expansion of $\boldsymbol{C}^{-1}$ and contains components of $\boldsymbol{C}_{0}$, arranged in a particular way.

The geometrical non-linearities are included in the matrices $\boldsymbol{B}, \boldsymbol{G}$ and $\tilde{\boldsymbol{S}}_{0} . \boldsymbol{B}$ represents the usual strain-displacement matrix, $\boldsymbol{G}$ relates the nodal displacements $\boldsymbol{u}$ and the gradient of displacements vector, and, finally, $\tilde{\boldsymbol{S}}_{0}$ represents a matrix that contains the initial stresses (we have chosen the same notation as in [17]).

In the right hand side of Eq. (35), the non linear load vector $\boldsymbol{f}_{p}^{n l}$ is a vector containing information of material and geometrical non-linearities of all order problems ranging from order one to $p-1$. It 
can be written as:

$$
\boldsymbol{f}_{p}^{n l}=\int_{\Omega_{0}}\left(\boldsymbol{B}^{T}\left(\boldsymbol{S}_{p}^{n l m a t}+\boldsymbol{S}_{p}^{n l g e o m}\right)+\boldsymbol{G}^{T} \boldsymbol{S}_{p}^{*}\right) d \Omega
$$

As in the stiffness matrix, $\boldsymbol{S}_{p}^{\text {nlgeom }}$ and $\boldsymbol{S}_{p}^{*}$ represent the standard matrices found in literature when ANM is used to solve geometrical non-linear problems with linear materials. $\boldsymbol{S}_{p}^{\text {nlmat }}$ takes into account the material behaviour:

$$
\begin{aligned}
\boldsymbol{S}_{p}^{n l m a t} & =\left(\lambda \ln J_{0}-\mu\right)\left(\boldsymbol{C} \boldsymbol{C}_{0}\left(R Z_{p}-\frac{R J_{p}}{J_{0}^{4}}\right)+\frac{\boldsymbol{R} \boldsymbol{C} \boldsymbol{C}_{p}}{J_{0}^{2}}+\boldsymbol{R} \boldsymbol{C}_{p}^{-1}\right) \\
& +\lambda\left(\frac{\boldsymbol{C} \boldsymbol{C}_{0}}{J_{0}^{2}}\left(R Y_{p}+\frac{R J_{p}}{2 J_{0}^{2}}\right)+\boldsymbol{R} \boldsymbol{S}_{p}\right)
\end{aligned}
$$

In this equation, $\boldsymbol{C} \boldsymbol{C}_{0}$ represents the cofactor matrix of $\boldsymbol{C}_{0}$ and $\boldsymbol{R C} \boldsymbol{C}_{p}$ is a vector containing values of $C_{i j}$ of all problems from order one to $p-1$, obtained when the cofactor matrix of $C$ is expanded in Taylor series:

$$
\begin{gathered}
\boldsymbol{C} \boldsymbol{C}_{p}=\tilde{\boldsymbol{C}}_{0} \boldsymbol{C}_{p}+\boldsymbol{R} \boldsymbol{C C}_{p}, \\
\boldsymbol{R} \boldsymbol{C} \boldsymbol{C}_{p}=\sum_{r=1}^{p-1}\left(\begin{array}{c}
C_{22}^{r} C_{33}^{p-r}-C_{23}^{r} C_{23}^{p-r} \\
C_{11}^{r} C_{33}^{p-r}-C_{13}^{r} C_{13}^{p-r} \\
C_{11}^{r} C_{22}^{p-r}-C_{12}^{r} C_{12}^{p-r} \\
C_{13}^{r} C_{23}^{p-r}-C_{12}^{r} C_{33}^{p-r} \\
C_{13}^{r} C_{12}^{p-r}-C_{11}^{r} C_{23}^{p-r} \\
C_{12}^{r} C_{23}^{p-r}-C_{13}^{r} C_{22}^{p-r}
\end{array}\right) .
\end{gathered}
$$

Here, $R J_{p}$ is a summation of products of different components of $\boldsymbol{C}_{p}$ and is obtained when the squared Jacobian is expanded in Taylor series:

$$
\left(J^{2}\right)_{p}=\boldsymbol{M}_{0}^{T} \boldsymbol{C}_{p}+R J_{p}
$$

$\boldsymbol{R} \boldsymbol{S}_{p}$ collects terms concerning the expansion of $Y=\ln J$ and $\boldsymbol{C}^{-1}$ :

$$
\boldsymbol{R} \boldsymbol{S}_{p}=\sum_{r=1}^{p-1} Y_{r} \boldsymbol{C}_{p-r}^{-1}
$$

$\boldsymbol{R} \boldsymbol{C}_{p}^{-1}$ collects terms concerning $Z=J^{-2}$ and cofactor matrix of $\boldsymbol{C}$ expansions:

$$
\boldsymbol{R} \boldsymbol{C}_{p}^{-1}=\sum_{r=1}^{p-1} Z_{r} \boldsymbol{C} \boldsymbol{C}_{p-r}
$$

Finally, it is necessary to expand $Y=\ln J$ and $Z=J^{-2}$ by using Taylor series and the chain rule generalized to higher derivatives:

$$
Y_{p}=\frac{1}{2 J_{0}^{2}}\left(J^{2}\right)_{p}+R Y_{p}, \text { and } Z_{p}=\frac{-1}{J_{0}^{4}}\left(J^{2}\right)_{p}+R Z_{p}
$$


where

$$
\begin{aligned}
R Y_{1} & =0 \\
R Y_{2} & \left.=\frac{-1}{4 J_{0}^{4}}\left(J^{2}\right)_{1}^{2}\right) \\
R Y_{3} & =\frac{1}{6 J_{0}^{6}}\left(J^{2}\right)_{1}^{3}+2 \frac{-1}{4 J_{0}^{4}}\left(J^{2}\right)_{1}\left(J^{2}\right)_{2}, \\
R Z_{1} & =0 \\
R Z_{2} & =\frac{1}{J_{0}^{6}}\left(J^{2}\right)_{1}^{2}, \\
R Z_{3} & =\frac{-1}{J_{0}^{8}}\left(J^{2}\right)_{1}^{3}+2 \frac{1}{J_{0}^{6}}\left(J^{2}\right)_{1}\left(J^{2}\right)_{2}, \\
\ldots &
\end{aligned}
$$

At this point, a procedure entirely similar to that of Eq. (36) is performed, leading to an equivalent expression of the displacement in terms of a power series in parameter $a$ where each term is composed by a finite sum of separable functions.

\section{REFERENCES}

1. H. Abichou, H. Zahrouni, and M. Potier-Ferry. Asymptotic numerical method for problems coupling several nonlinearities Computer Methods in Applied Mechanics and Engineering, 191(51-52):5795-5810, 2002.

2. Jérémie Allard, Stéphane Cotin, François Faure, Pierre-Jean Bensoussan, François Poyer, Christian Duriez, Hervé Delingette, and Laurent Grisoni. SOFA an Open Source Framework for Medical Simulation. In Medicine Meets Virtual Reality (MMVR'15), Long Beach, USA, February 2007.

3. A. Ammar, F. Chinesta, P. Diez, and A. Huerta. An error estimator for separated representations of highly multidimensional models. Computer Methods in Applied Mechanics and Engineering, 199(25-28):1872 - 1880, 2010.

4. A. Ammar, B. Mokdad, F. Chinesta, , and R. Keunings. A new family of solvers for some classes of multidimensional partial differential equations encountered in kinetic theory modeling of complex fluids. part ii: transient simulation using space-time separated representations. J. Non-Newtonian Fluid Mech., 144:98-121, 2007.

5. A. Ammar, B. Mokdad, F. Chinesta, and R. Keunings. A new family of solvers for some classes of multidimensional partial differential equations encountered in kinetic theory modeling of complex fluids. J. Non-Newtonian Fluid Mech., 139:153-176, 2006.

6. D. Amsallem and Ch. Farhat. An Interpolation Method for Adapting Reduced-Order Models and Application to Aeroelasticity. AIAA Journal, 46:1803-1813, 2008.

7. Jernej Barbič and Doug L. James. Real-time subspace integration for St. Venant-Kirchhoff deformable models. $A C M$ Transactions on Graphics (SIGGRAPH 2005), 24(3):982-990, August 2005.

8. M Barrault, Y Maday, NC Nguyen, and AT Patera. An 'empirical interpolation' method: application to efficient reducedbasis discretization of partial differential equations. Comptes Rendus Mathematique, 339(9):667-672, NOV 12004.

9. B. Bognet, F. Bordeu, F. Chinesta, A. Leygue, and A. Poitou. Advanced simulation of models defined in plate geometries: 3d solutions with 2d computational complexity. Computer Methods in Applied Mechanics and Engineering, 201-204(0):1 $-12,2012$.

10. J. Bonet and R. D. Wood. Nonlinear continuum mechanics for finite element analysis. Cambridge University Press, 2008.

11. M. Bro-Nielsen and S. Cotin. Real-time volumetric deformable models for surgery simulation using finite elements and condensation. Computer Graphics Forum, 15(3):57-66, 1996.

12. H.-L. Cao and M. Potier-Ferry. An improved iterative method for large strain viscoplastic problems. International Journal for Numerical Methods in Engineering, 44:155-176, 1999.

13. Saifon Chaturantabut and Danny C. Sorensen. Nonlinear model reduction via discrete empirical interpolation. SIAM J. Sci. Comput., 32:2737-2764, September 2010.

14. F. Chinesta, A. Ammar, and E. Cueto. Recent advances in the use of the Proper Generalized Decomposition for solving multidimensional models. Archives of Computational Methods in Engineering, 17(4):327-350, 2010. 
15. Francisco Chinesta, Pierre Ladeveze, and Elias Cueto. A short review on model order reduction based on proper generalized decomposition. Archives of Computational Methods in Engineering, 18:395-404, 2011.

16. B. Cochelin, N. Damil, and M. Potier-Ferry. Asymptotic-numerical methods and Padé approximants for non-linear elastic structures. International Journal for Numerical Methods in Engineering, 37:1187-1213, 1994.

17. B. Cochelin, N. Damil, and M. Potier-Ferry. The asymptotic numerical method: an efficient perturbation technique for nonlinear structural mechanics. Revue Europeenne des Elements Finis, 3:281-297, 1994.

18. H. Delingette and N. Ayache. Soft tissue modeling for surgery simulation. In N. Ayache, editor, Computational Models for the Human Body, Handbook of Numerical Analysis (Ph. Ciarlet, Ed.), pages 453-550. Elsevier, 2004.

19. Ch. Ghnatios, F. Chinesta, E. Cueto, A. Leygue, A. Poitou, P. Breitkopf, and P. Villon. Methodological approach to efficient modeling and optimization of thermal processes taking place in a die: Application to pultrusion. Composites Part A: Applied Science and Manufacturing, 42(9):1169 - 1178, 2011.

20. Ch. Ghnatios, F. Masson, A. Huerta, A. Leygue, E. Cueto, and F. Chinesta. Proper generalized decomposition based dynamic data-driven control of thermal processes. Computer Methods in Applied Mechanics and Engineering, 213216(0):29-41, 2012.

21. D. Gonzalez, F. Masson, F. Poulhaon, E. Cueto, and F. Chinesta. Proper generalized decomposition based dynamic data driven inverse identification. Mathematics and Computers in Simulation, in press, 2012.

22. S. R. Idelsohn and R. Cardona. A reduction method for nonlinear structural dynamics analysis. Computer Methods in Applied Mechanics and Engineering, 49:253-279, 1985.

23. K. Karhunen. Uber lineare methoden in der wahrscheinlichkeitsrechnung. Ann. Acad. Sci. Fennicae, ser. Al. Math. Phys., 37, 1946.

24. P. Krysl, S. Lall, and J.E. Marsden. Dimensional model reduction in non-linear finite element dynamics of solids and structures. Int. J. Numer. Meth. in Engng., 51:479-504, 2001.

25. P. Ladeveze. Nonlinear Computational Structural Mechanics. Springer, N.Y., 1999.

26. P. Ladeveze, J.-C. Passieux, and D. Neron. The latin multiscale computational method and the proper generalized decomposition. Computer Methods in Applied Mechanics and Engineering, 199(21-22):1287 - 1296, 2010.

27. Pierre Ladeveze and Ludovic Chamoin. On the verification of model reduction methods based on the proper generalized decomposition. Computer Methods in Applied Mechanics and Engineering, 200(23-24):2032-2047, 2011.

28. C. Le Bris, T. Lelièvre, and Y. Maday. Results and questions on a nonlinear approximation approach for solving highdimensional partial differential equations. Constructive Approximation, 30:621-651, 2009. 10.1007/s00365-009-9071-1.

29. M. M. Loève. Probability theory. The University Series in Higher Mathematics, 3rd ed. Van Nostrand, Princeton, NJ, 1963.

30. E. N. Lorenz. Empirical Orthogonal Functions and Statistical Weather Prediction. MIT, Departement of Meteorology, Scientific Report Number 1, Statistical Forecasting Project, 1956.

31. N. C. Nguyen, A. T. Patera, and J. Peraire. A 'best points' interpolation method for efficient approximation of parametrized functions. International Journal for Numerical Methods in Engineering, 73(4):521-543, JAN 222008.

32. S. Niroomandi, I. Alfaro, E. Cueto, and F. Chinesta. Real-time deformable models of non-linear tissues by model reduction techniques. Computer Methods and Programs in Biomedicine, 91(3):223 - 231, 2008.

33. S. Niroomandi, I. Alfaro, E. Cueto, and F. Chinesta. Accounting for large deformations in real-time simulations of soft tissues based on reduced-order models. Computer Methods and Programs in Biomedicine, 105(1):1-12, 2012.

34. S. Niroomandi, D. Gonzalez, I. Alfaro, F. Bordeu, A. Leygue, E. Cueto, and F. Chinesta. Real time simulation of biological soft tissues: a PGD approach. International Journal for Numerical Methods in Biomedical Engineering, submitted, 2012.

35. Siamak Niroomandi, Iciar Alfaro, Elias Cueto, and Francisco Chinesta. Model order reduction for hyperelastic materials. International Journal for Numerical Methods in Engineering, 81(9):1180-1206, 2010.

36. Anthony Nouy. A priori model reduction through Proper Generalized Decomposition for solving time-dependent partial differential equations. Computer Methods in Applied Mechanics and Engineering, 199(23-24):1603-1626, 2010.

37. H. M. Park and D. H. Cho. The use of the Karhunen-Loève decomposition for the modeling of distributed parameter systems. Chemical Engineering Science, 51(1):81-98, 1996.

38. E. Pruliere, F. Chinesta, and A. Ammar. On the deterministic solution of multidimensional parametric models using the Proper Generalized Decomposition. Mathematics and Computers in Simulation, 81(4):791-810, DEC 2010.

39. N. Relun, D. Neron, and P.-A. Boucard. A model reduction technique based on the pgd for elastic-viscoplastic computational analysis. Computational Mechanics, 51:83-92, 2013.

40. D. Ryckelynck. A priori model reduction method for the optimization of complex problems. In Workshop on Optimal Design of Materials and Structures, Ecole Polytechnique, Palaiseau, Paris (France), 2003.

41. D. Ryckelynck. A priori hyperreduction method: an adaptive approach. Journal of Computational Physics, 202(1):346$366,2005$.

42. D. Ryckelynck, F. Chinesta, E. Cueto, and A. Ammar. On the a priori Model Reduction: Overview and recent developments. Archives of Computational Methods in Engineering, 12(1):91-128, 2006.

43. Z.A. Taylor, S. Crozier, and S. Ourselin. A reduced order explicit dynamic finite element algorithm for surgical simulation. Medical Imaging, IEEE Transactions on, 30(9):1713 -1721, sept. 2011.

44. Z.A. Taylor, S. Ourselin, and S. Crozier. A reduced order finite element algorithm for surgical simulation. In Engineering in Medicine and Biology Society (EMBC), 2010 Annual International Conference of the IEEE, pages 239-242, $312010-$ 
sept. 42010 .

45. J. B. Tenenbaum, V. de Silva, and J. C. Langford. A global framework for nonlinear dimensionality reduction. Science, 290:2319-2323, 2000.

46. J. Yvonnet, H. Zahrouni, and M. Potier-Ferry. A model reduction method for the post-buckling analysis of cellular microstructures . Computer Methods in Applied Mechanics and Engineering, 197:265-280, 2007. 